\title{
Cortical and Thalamic Excitation Mediate the Multiphasic Responses of Striatal Cholinergic Interneurons to Motivationally Salient Stimuli
}

\author{
Natalie M. Doig, ${ }^{1}$ Peter J. Magill, ${ }^{1}$ Paul Apicella, ${ }^{2}$ J. Paul Bolam, ${ }^{1}$ and Andrew Sharott ${ }^{1}$ \\ ${ }^{1}$ Medical Research Council Anatomical Neuropharmacology Unit, Department of Pharmacology, University of Oxford, Oxford OX1 3TH, United Kingdom; \\ and 'Institut de Neurosciences de la Timone, Centre National de la Recherche Scientifique-Aix-Marseille Université, 13005 Marseille, France
}

\begin{abstract}
Cholinergic interneurons are key components of striatal microcircuits. In primates, tonically active neurons (putative cholinergic interneurons) exhibit multiphasic responses to motivationally salient stimuli that mirror those of midbrain dopamine neurons and together these two systems mediate reward-related learning in basal ganglia circuits. Here, we addressed the potential contribution of cortical and thalamic excitatory inputs to the characteristic multiphasic responses of cholinergic interneurons in vivo. We first recorded and labeled individual cholinergic interneurons in anesthetized rats. Electron microscopic analyses of these labeled neurons demonstrated that an individual interneuron could form synapses with cortical and, more commonly, thalamic afferents. Single-pulse electrical stimulation of ipsilateral frontal cortex led to robust short-latency $(<20 \mathrm{~ms})$ interneuron spiking, indicating monosynaptic connectivity, but firing probability progressively decreased during high-frequency pulse trains. In contrast, single-pulse thalamic stimulation led to weak short-latency spiking, but firing probability increased during pulse trains. After initial excitation from cortex or thalamus, interneurons displayed a "pause" in firing, followed by a "rebound" increase in firing rate. Across all stimulation protocols, the number of spikes in the initial excitation correlated positively with pause duration and negatively with rebound magnitude. The magnitude of the initial excitation, therefore, partly determined the profile of later components of multiphasic responses. Upon examining the responses of tonically active neurons in behaving primates, we found that these correlations held true for unit responses to a reward-predicting stimulus, but not to the reward alone, delivered outside of any task. We conclude that excitatory inputs determine, at least in part, the multiphasic responses of cholinergic interneurons under specific behavioral conditions.
\end{abstract}

Key words: basal ganglia; corticostriatal; parafascicular nucleus; thalamostriatal; tonically active neuron

\section{Introduction}

The striatum is the principal site of integration of cortical and thalamic information in the basal ganglia. Although mediumsized spiny projection neurons (MSNs) are the main target of both cortical and thalamic projections (Somogyi et al., 1981; Dubé et al., 1988; Doig et al., 2010), certain types of interneurons are selectively targeted by cortical and/or thalamic afferents, so these glutamatergic inputs can powerfully influence the output of the striatum (Mallet et al., 2005; Ding et al., 2010). Cholinergic

Received Oct. 28, 2013; revised Jan. 17, 2014; accepted Jan. 23, 2014.

Author contributions: N.M.D., P.J.M., J.P.B., and A.S. designed research; N.M.D., P.A., and A.S. performed research; A.S. analyzed data; N.M.D. and A.S. wrote the paper.

This work was supported by the Medical Research Council UK (Grants U138197109 and U138164490), Parkinson's UK (Grant G-0806), a Marie Curie European Re-integration Grant (SNAP-PD) awarded by the European Union, and an International Joint Project Grant (Grant JP090457) awarded by The Royal Society. N.M.D. was in receipt of a Medical Research Council studentship. We thank J. Morgan for the generous gift of antibodies against Cerebellin-1; G. Hazell, B. Micklem, L. Norman, K. Whitworth, and C. Johnson for technical support; I. Huerta-Ocampo and K. Nakamura for advice and assistance with immunohistochemistry; F. Vinciati for contributing to electrophysiological recordings; members of the Apicella laboratory for help with TAN data collection; and P. Henny for assistance with EM analysis and helpful comments on the manuscript.

Correspondence should be addressed to Andrew Sharott, MRC Anatomical Neuropharmacology Unit, Department of Pharmacology, Mansfield Road, Oxford 0X1 3TH, United Kingdom. E-mail: andrew.sharott@pharm.ox.ac.uk.

DOl:10.1523/JNEUROSCI.4627-13.2014

Copyright $\odot 2014$ the authors $\quad 0270-6474 / 14 / 343101-17 \$ 15.00 / 0$ interneurons are prime candidates for mediating the effects of excitatory inputs to striatum and have been studied extensively in relation to reinforcement learning through their putative identification as "tonically active neurons" (TANs) in behaving animals. TANs are particularly sensitive to motivationally salient stimuli, usually responding with a pause in their tonic firing that can be preceded by short-latency firing and/or followed by a "rebound" increase in spiking (Morris et al., 2004; Joshua et al., 2008). The timing of these responses is entwined with those of midbrain dopamine neurons to the same stimuli that encode reward value (Morris et al., 2004). These coordinated systems modulate the timing of reinforcement learning in the striatum and thus shape the behavioral output of the basal ganglia (Apicella, 2007).

The mechanisms underlying the responses of cholinergic interneurons to motivationally salient stimuli are unclear. GABAergic inputs from MSNs (Gonzales et al., 2013) could initiate the pause, but physiological evidence for such a connection is lacking. Therefore, GABAergic interneurons have also been implicated (Sullivan et al., 2008). Furthermore, the intrinsic membrane properties and autonomous firing of cholinergic neurons mean that a brief barrage of synaptic excitation can produce complex changes in their firing patterns, including pauses in continuous firing (Oswald et al., 2009; Ding et al., 2010). Both ana- 
tomical (Lapper and Bolam, 1992) and physiological (Ding et al., 2010; Threlfell et al., 2012) evidence suggests that a major source of this synaptic excitation are the projections from the intralaminar thalamic nuclei (ITN). Indeed, thalamic input appears necessary for the pause response of TANs in nonhuman primates (Matsumoto et al., 2001). In vivo, electrical stimulation of cortex has also been shown to excite cholinergic interneurons at short latencies, suggesting a monosynaptic connection (Reynolds and Wickens, 2004; Sharott et al., 2012), but there is little anatomical evidence to suggest direct cortical innervation (Lapper and Bolam, 1992; Dimova et al., 1993).

In this study, we demonstrate that the same cholinergic interneuron can form synapses with both cortical and thalamic afferents and that driving these excitatory synapses can lead to distinctive effects on interneuron firing properties. The magnitude of the initial excitation in these interneurons predicts the temporal profile of the subsequent response components. Finally, we show that this correlation between initial excitation and the profile of the multiphasic response is present in TANs responding to specific motivationally relevant stimuli in behaving primates.

\section{Materials and Methods}

Electrophysiological recordings in rats

Experimental procedures were performed on adult male Sprague Dawley rats (Charles River Laboratories) and were conducted in accordance with the Animals (Scientific Procedures) Act of 1986 (United Kingdom) and with Society for Neuroscience Policies on the Use of Animals in Neuroscience Research.

Recording and labeling experiments were performed in 27 anesthetized rats $(280-340 \mathrm{~g})$. Briefly, anesthesia was induced with $4 \% \mathrm{v} / \mathrm{v}$ isoflurane in $\mathrm{O}_{2}$, and maintained with urethane $(1.3 \mathrm{~g} / \mathrm{kg}$ ethyl carbamate, i.p.; Sigma-Aldrich) and supplemental doses of ketamine $(30 \mathrm{mg} / \mathrm{kg} \mathrm{Ke}-$ taset, i.p.; Willows Francis) and xylazine (3 mg/kg Rompun, i.p.; Bayer), as described previously (Magill et al., 2006; Sharott et al., 2012). Wound margins were infiltrated with local anesthetic $(0.5 \% \mathrm{w} / \mathrm{v}$ bupivacaine; AstraZeneca). Animals were then placed in a stereotaxic frame (Kopf). Body temperature was maintained at $37 \pm 0.5^{\circ} \mathrm{C}$ with a homoeothermic heating device (Harvard Apparatus). Electrocorticogram (ECoG) readings, electrocardiographic activity, and respiration rate were monitored constantly to ensure the animals' wellbeing. The epidural ECoG was recorded above the frontal (somatic sensory-motor) cortex $(4.2 \mathrm{~mm}$ anterior and $2.0 \mathrm{~mm}$ lateral of bregma; Paxinos and Watson, 1986) and was referenced against the ipsilateral cerebellar hemisphere (Mallet et al., 2008). Raw ECoG was band-pass filtered $(0.3-1500 \mathrm{~Hz},-3 \mathrm{~dB}$ limits) and amplified (2000×; DPA-2FS filter/amplifier; NPI Electronic) before acquisition. Extracellular recordings of the action potentials ("spikes") of individual neurons (i.e., single-unit activities) in the dorsal striatum were made using glass electrodes (10-30 $\mathrm{M} \Omega$ in situ; tip diameter $\sim 1.2 \mu \mathrm{m}$ ) containing $0.5 \mathrm{M} \mathrm{NaCl}$ solution and neurobiotin (NB; $1.5 \% \mathrm{w} / \mathrm{v}$; Vector Laboratories). Electrodes were lowered into the brain under stereotaxic guidance and using a computer-controlled stepper motor (IVM-1000; Scientifica) that allowed the electrode depth to be determined with a resolution of $0.1 \mu \mathrm{m}$. Electrode signals were amplified 10 times through the bridge circuitry of an Axoprobe-1A amplifier (Molecular Devices), AC coupled, amplified another 100 times, and band-pass filtered at $300-$ $5000 \mathrm{~Hz}$ (DPA-2FS filter/amplifier). The ECoG and single-unit activity were each sampled at $16.6 \mathrm{kHz}$ using a Power1401 analog-digital converter and a PC running Spike2 acquisition and analysis software (Version 7.2; Cambridge Electronic Design). After electrophysiological recordings, single neurons were juxtacellularly labeled with NB (Magill et al., 2000). Briefly, positive current pulses (2-10 nA, $200 \mathrm{~ms}, 50 \%$ duty cycle) were applied until the single-unit activity became robustly entrained by the current injections. After the recording and labeling sessions, the animals were given a lethal dose of ketamine $(150 \mathrm{mg} / \mathrm{kg})$ and were perfused transcardially with $100 \mathrm{ml}$ of $0.05 \mathrm{M}$ PBS, pH 7.4, followed by $300 \mathrm{ml}$ of $0.1 \% \mathrm{w} / \mathrm{v}$ glutaraldehyde and $4 \% \mathrm{w} / \mathrm{v}$ paraformaldehyde in $0.1 \mathrm{~m}$ phosphate buffer, $\mathrm{pH} 7.4(\mathrm{~PB})$, and then by $200 \mathrm{ml}$ of $4 \% \mathrm{w} / \mathrm{v}$ paraformaldehyde in PB. Brains were then left in the latter fixative solution at $4^{\circ} \mathrm{C}$ until sectioning $24-72 \mathrm{~h}$ later.

Electrical stimulation of the cortex and thalamus. Parallel, bipolar stimulating electrodes (constructed from nylon-coated stainless steel wires; California Fine Wire) with tip diameters of $\sim 100 \mu \mathrm{m}$, a tip separation of $\sim 150 \mu \mathrm{m}$, and an impedance of $\sim 10 \mathrm{k} \Omega$ were implanted into the ipsilateral frontal cortex (Magill et al., 2004) and ipsilateral intralaminar thalamus. The coordinates of the cortical stimulation sites $(2.5-4.0 \mathrm{~mm}$ anterior and 2.5-3.0 mm lateral of bregma, at depths of $2.0-2.2 \mathrm{~mm}$ below the dura) correspond to layers $5 / 6$ of the primary/secondary motor cortices (Paxinos and Watson, 1986). For thalamic stimulation, we used coordinates ( $3.7 \mathrm{~mm}$ posterior and $1.3 \mathrm{~mm}$ lateral of bregma, at depths of $5.4-5.7 \mathrm{~mm}$ below the dura) that consistently led to an electrode track in the parafascicular nucleus (Pfn). Electrical stimuli, which consisted of square-wave current pulses of $0.3 \mathrm{~ms}$ duration and variable amplitude (100-900 $\mu \mathrm{A}$ ), were delivered using a constant-current isolator (A360D; World Precision Instruments) that was gated by digital outputs from the Power1401 converter. Different stimulation protocols were applied to cortex and then thalamus or vice versa depending on the response of the individual neuron: (1) a single-pulse stimulation delivered to cortex/ thalamus, (2) a paired-pulse stimulus given with a $100 \mathrm{~ms}$ interstimulus interval, and (3) a train of 5 stimuli given with a $25 \mathrm{~ms}$ interstimulus interval. Regardless of the type of stimulation (single, paired, or $40 \mathrm{~Hz}$ train), each was given at $2 \mathrm{~s}$ intervals.

Tissue processing for identification of recorded and labeled neurons. Parasagittal sections $(50 \mu \mathrm{m})$ were cut from each brain using a vibrating microtome (VT1000S; Leica Microsystems), collected in series, and washed in PBS. Free-floating sections were then incubated overnight at room temperature in Triton PBS (PBS containing 0.3\% v/v Triton X-100; Sigma-Aldrich) containing Cy3-conjugated streptavidin (1:1000 dilution; Life Technologies). Sections containing NB-labeled neuronal somata (those marked with Cy3) were then isolated for molecular characterization by indirect immunofluorescence (Sharott et al., 2012). Neurons with densely spiny higher-order dendrites were classified as projection neurons (MSNs) and were thus not tested for interneuron markers. Aspiny neurons were tested for expression of immunoreactivity for choline acetyltransferase (ChAT). Briefly, after 1-2 h of incubation in Triton PBS containing 10\% v/v normal donkey serum (NDS; Jackson ImmunoResearch Laboratories), sections containing NB-labeled neuronal somata were incubated in Triton PBS containing 2\% v/v NDS and a primary antibody against ChAT overnight at room temperature (goatanti-ChAT, catalog \# AB144P, 1:500; Millipore Bioscience Research Reagents). A fluorescent secondary antibody was then applied (Alexa Fluor 488; donkey-anti-goat; 1:000; Jackson ImmunoResearch) for $\sim 4$ h. Only NB-labeled neurons expressing ChAT were included in this study. To determine the placement of the thalamic stimulating electrode, an antibody against cerebellin 1 (rabbit anti-CBLN 1, 1:3000; a gift from J. Morgan, St. Jude Children's Research Hospital) with heat pretreatment as a means of antigen retrieval (Jiao et al., 1999) was used on sections in which the electrode track was apparent. Cerebellin 1 is a selective marker of the Pfn compared with neighboring structures (Kusnoor et al., 2010); in some cases, this was combined with Nissl staining to delineate the thalamic nuclei (NeuroTrace, catalog \#N21479, 1:100; Invitrogen). Judging from the robust responses of interneurons that we obtained and the anatomical verifications, it is likely that both poles of the stimulating electrodes were located in, or very close to, the Pfn. Despite this, we cannot rule out the possibility that the evoked electric field was not confined to the Pfn, so we conservatively refer to stimulation as being "thalamic."

\section{Electron microscopy}

Immunohistochemistry. Four recorded and labeled cholinergic interneurons from four rats were processed for electron microscopy (EM). The first neuron was a pilot study to establish the immunohistochemical and electron microscopic protocols. The four neurons used for EM analysis were positive for ChAT. Sections containing neuronal processes (lateral and medial to the section containing the soma) were isolated for EM processing. Sections were incubated in cryoprotectant $(25 \%$ sucrose, $10 \%$ glycerol) overnight at $4^{\circ} \mathrm{C}$. Sections (one at a time) were then freeze 
thawed using liquid nitrogen. Each section was laid flat in one well of a six-well plate and excess cryoprotectant was removed using filter paper. The well plate was then lowered over liquid nitrogen until the section froze and went opaque. The section was then rapidly thawed using cryoprotectant that had been kept at room temperature. The sections were then incubated in cryoprotectant for at least $1 \mathrm{~h}$ at room temperature or until all sections had sunk to the bottom of the glass vial. The freezing process was then repeated for all of the sections and the sections were thawed with room temperature $0.1 \mathrm{M} \mathrm{PB}$. All of the sections were then washed in $0.1 \mathrm{M} \mathrm{PB}$ twice and three times in PBS and incubated in PBS containing $10 \%$ NDS for $1 \mathrm{~h}$ at room temperature on a shaker. The sections were then incubated in avidin-biotin-peroxidase complex (ABC, 1:250; Vector Labs) prepared according to the manufacturer's instructions in a $1 \%$ NDS PBS solution.

After incubation in $\mathrm{ABC}$, the sections were washed 3 times in PBS, followed by 3 washes in $0.1 \mathrm{M} \mathrm{PB}, \mathrm{pH}$ 6.0. A peroxidase reaction using tetramethylbenzidine (TMB) as the chromogen was then performed to reveal the NB-filled neuron (Doig et al., 2010). Sections were placed for $20 \mathrm{~min}$ in a preincubation solution containing the following: $80 \mathrm{ml}$ of 0.1 M PB, pH 6.0, $4 \mathrm{ml}$ of ammonium paratungstate $\left(1 \%\right.$ in deionized $\left.\mathrm{H}_{2} \mathrm{O}\right)$, $1 \mathrm{ml}$ of TMB (catalog \# T5525, 0.2\% dissolved in absolute ethanol; Sigma-Aldrich), $800 \mu \mathrm{l}$ of ammonium chloride $(0.4 \%$ in deionized $\mathrm{H}_{2} \mathrm{O}$ ), and $800 \mu \mathrm{l}$ of D-glucose ( $20 \%$ in deionized $\mathrm{H}_{2} \mathrm{O}$ ). To perform the reaction, the preincubation solution was removed and replaced with the reaction solution, which consisted of $2 \mathrm{ml}$ of the preincubation solution (as above) plus $2 \mu \mathrm{l}$ of glucose oxidase (catalog \#G6891; Sigma-Aldrich). The reaction was initially performed on the section containing the cell body to gauge the optimum processing time; the section was wet mounted and checked under a light microscope $(40 \times)$. The reaction was then allowed to progress for all sections for between 6 and 8 min depending on the neuron. The reaction was stopped with $0.1 \mathrm{M} \mathrm{PB}, \mathrm{pH}$ 6.0. Sections were then washed 3 times for $5 \mathrm{~min}$ with $0.1 \mathrm{M} \mathrm{PB}, \mathrm{pH}$ 6.0. The reaction was then stabilized by incubation in a solution containing the following: $400 \mu \mathrm{l}$ of ammonium chloride $\left(0.4 \%\right.$ in deionized $\left.\mathrm{H}_{2} \mathrm{O}\right), 400$ $\mu \mathrm{l}$ of D-glucose ( $20 \%$ in deionized $\left.\mathrm{H}_{2} \mathrm{O}\right), 800 \mu \mathrm{l}$ of cobalt (II) chloride ( $1 \%$ in deionized $\mathrm{H}_{2} \mathrm{O}$ ), and $40 \mathrm{mg}$ of DAB dissolved in $40 \mathrm{ml}$ of $0.1 \mathrm{M} \mathrm{PB}$, $\mathrm{pH}$ 6.0. The stabilization solution was filtered before use. Sections were incubated for $15 \mathrm{~min}$ at room temperature. The stabilization solution was then removed and replaced with 310 min washes of $0.1 \mathrm{M} \mathrm{PB}, \mathrm{pH}$ 6.0. During stabilization, the blue-staining color from the reaction step changes to a darker magenta color and background staining is reduced. Sections were then washed 3 times in $0.1 \mathrm{M} \mathrm{PB}, \mathrm{pH}$ 7.4.

Alternate sections were then processed to reveal either VGluT1 or VGluT2 labeling using the peroxidase-anti-peroxidase (PAP) method (Bolam, 1992). Alternate sections were incubated in a primary antibody against either VGluT1 (rabbit-anti-VGluT1, catalog \#VGT3, 1:2000; MAb Tech) or VGluT2 (rabbit-anti-VGluT2, catalog \#, 135403, 1:2000; Synaptic Systems) overnight at room temperature in PBS. The sections were then incubated in an un-conjugated antibody (donkey-anti-rabbit IgG, catalog \#711-035-152, 1:100; Jackson ImmunoResearch) for at least $4 \mathrm{~h}$ at room temperature. Next, the sections were incubated in a PAP complex (rabbit PAP, catalog \#323-005-024, 1:400; Jackson ImmunoResearch) for at least $4 \mathrm{~h}$ at room temperature. A peroxidase reaction using $\mathrm{DAB}$ as the chromogen was used to reveal VGluT1 or VGlut2 labeling. Sections were incubated in a solution containing DAB $(0.05 \%)$ dissolved in TRIS buffer for $20 \mathrm{~min}$. Hydrogen peroxidase $\left(\mathrm{H}_{2} \mathrm{O}_{2}\right)$ was then added to the solution to achieve a final concentration of $0.01 \%$. The reaction was monitored using a dissection microscope. All sections were incubated for between 3 and $4 \mathrm{~min}$. They were then washed in Tris buffer 3 times, followed by 3 washes in $0.1 \mathrm{M} \mathrm{PB}, \mathrm{pH}$ 7.4.

Sections were then post-fixed in osmium tetroxide $(1 \% \mathrm{w} / \mathrm{v}$ in $\mathrm{PB}$; Oxkem) for $25 \mathrm{~min}$ and then washed in $0.1 \mathrm{M} \mathrm{PB}$ and dehydrated in an ascending series of ethanol dilutions as follows: $15 \mathrm{~min}$ in $50 \% \mathrm{w} / \mathrm{v}$ ethanol, $35 \mathrm{~min}$ in $70 \%$ ethanol that included $1 \% \mathrm{w} / \mathrm{v}$ uranyl acetate, $15 \mathrm{~min}$ in $95 \%$ ethanol, and twice for $15 \mathrm{~min}$ in absolute ethanol. After absolute ethanol, sections were washed twice in propylene oxide (Sigma-Aldrich) for $15 \mathrm{~min}$ and placed into resin (Durcupan ACM; Fluka) and left overnight at room temperature. Sections were then placed on microscope slides, a coverslip was applied, and the resin was cured at $65^{\circ} \mathrm{C}$ for $\sim 72 \mathrm{~h}$.
Reconstruction and ultrathin sectioning. The 3D reconstruction of NBlabeled neurons was performed using Neurolucida (MBF) using an oilimmersion objective $(60 \times)$. The tracings within the separate sections were then spliced using Neurolucida and the final version was corrected for shrinkage in all dimensions: $x(6.3 \%), y(6.0 \%)$, and $z$ (8\%; Sadek et al., 2007). A file with the entire neuron unspliced was saved to be used as a guide for resectioning dendritic fragments. Quantitative data from the reconstructions was then obtained using Neuroexplorer (MBF). For the Sholl analysis, radius segments of $50 \mu \mathrm{m}$ were used. The data were then exported to Microsoft Excel (2007).

Light microscopic images of the dendrites of each neuron $(20 \times)$ were aligned with the reconstruction to identify specific dendritic fragments in each sagittal section. At least one dendritic fragment was reembedded from each sagittal section from the "top" of the section. Ultrathin sections $(50 \mathrm{~nm})$ containing the pieces of dendrite were then resectioned using an ultramicrotome (EM UC6; Leica Microsystems) and collected on pioloform-coated single slot grids. The sections were then contrasted using lead citrate.

Electron microscopy and analysis. The dendritic fragment(s) within each ultrathin section was then imaged using an electron microscope (CM10; Philips) at a high magnification $(13,500 \times$ to $46,000 \times)$. Each dendritic fragment was analyzed through several grids and in serial section on each grid, with a minimum of five sections analyzed per grid. The major factor limiting the extent of dendrite that could be analyzed was the penetration of the VGluT antibodies. To control for the possibility of VGluT-false-negative terminals, serial sections on a grid were only examined if there was at least one VGluT1/2-positive profile present in one of the sections on the grid (at a magnification of at least 13,500×).

Electron micrographs were then analyzed using ImageJ software (Version 1.41o). Every synapse formed with the sections of dendrites examined was analyzed and every presynaptic terminal forming a symmetric (type II) or an asymmetric (type I) synapse with the labeled dendrite was recorded. The length of the postsynaptic density for every synapse formed with the cholinergic dendrite was measured. Synaptic density was calculated as the number of synapses divided by the length of dendrite (in micrometers) examined (in the $z$-axis, from the number of ultrathin sections analyzed) and then expressed as density per $10 \mu \mathrm{m}$.

Estimations of the total number of synapses onto the dendrites of cholinergic interneurons were calculated as follows, based on data acquired. To estimate the total number of synapses, the average height examined for each section was calculated based on the number of ultrathin sections examined and the number of synapses found within that height was obtained from the data. For example, for neuron NJX009, the average height per section examined was $2.12 \mu \mathrm{m}$ (out of a potential 45 $\mu \mathrm{m}$ of section thickness) and a total of 90 synapses (symmetric, asymmetric, and VGluT2 positive) were counted within this thickness. The number of synapses was then multiplied by a correction factor to estimate the total number of synapses as if the entire height of the section was analyzed. For NJX009, there were 12 sections, each with a thickness of 45 $\mu \mathrm{m}$ (after shrinkage), so the number of synapses (90) was thus multiplied by $20.2(=1818.13)$. The total number of high endings of dendrite for each neuron was counted from the unspliced Neurolucida files. For NJX009, a possible total of 78 high endings could have been sampled and, out of this, 20 were examined. A correction factor was then used to estimate what the number of synapses would be had every high ending been sampled. In the case of NJX009, this correction factor was 3.9, so the predicted number of synapses for NJX009 is $7090.70\left(1818.13^{*} 3.9\right)$. This method is based on the protocol established by Henny et al. $(2012,2013)$. We chose to use this method because it is based entirely on the EM ultrastructural data and does not take into account dendritic length, which decreases the error, for the following reasons: (1) the dendritic length would be estimated from the Neurolucida files (and not the ultrathin sections) and (2) by using this method, the orientation of the dendrite within the section is not incorporated. The method was then repeated to estimate the total number of symmetric, asymmetric, and VGluT1 or VGluT2 synapses for each neuron. Numbers of VGluT1 and VGluT2 synapses were doubled to take into account the fact that the sampling was only performed in one of two series. 


\section{Electrophysiological recordings of TANs in behaving primates}

Single neurons were recorded from the striatum of four behaving macaque monkeys (identified as C, P, G, and B). The monkeys were seated in a restraining box that was described previously (Apicella et al., 1997) and faced a panel placed $\sim 30 \mathrm{~cm}$ in front of them. Each was chronically fitted with a head-restraining device and a recording chamber over a craniotomy for electrode insertions mainly targeted at the putamen. Surgical and electrophysiological procedures were as described previously (Apicella et al., 1997). Monkey surgery and behavioral testing protocols were in accordance with guidelines set by the National Institutes of Health and the French government regulations on animal experimentation. Neurons were accessed on vertical penetrations with glass-coated tungsten electrodes. The electrodes were advanced with a hydraulic microdrive (MO-95; Narishige) through a stainless steel guide tube that was used to penetrate the dura. Signals from neuronal activity were conventionally amplified, filtered (band pass, $0.3-1.5 \mathrm{kHz}$ ), and converted to digital pulses through a window discriminator. Putative cholinergic interneurons (TANs) were classified according to their electrophysiological characteristics, as described previously (Aosaki et al., 1994), as well as their typical responses to unexpected rewarding stimuli (Apicella et al., 1997). Our focus on TANs recorded in putamen was not only in keeping with many other past studies of salience-/reward-related TAN activity in behaving primates (Aosaki et al., 1994; Apicella et al., 1997; Morris et al., 2004), but also allowed for the most direct comparison (in terms of broadly equivalent circuits and function) with our sample of rat cholinergic interneurons. Although there is some regional bias in the responses of primate striatal neurons to salient/rewarding stimuli, uniformity in TAN responses across the striatal axis has been commonly emphasized (Morris et al., 2004; Adler et al., 2013a; Adler et al., 2013b).

The activity of TANs were studied with respect to two behavioral conditions. In the first condition, a Pavlovian protocol in which the onset of a visual stimulus on the center of the panel at unpredicted times was followed, after a $1 \mathrm{~s}$ delay, by the delivery of liquid reward $(0.3 \mathrm{ml}$ of apple juice) via a tube positioned directly in front of the monkey's mouth. All monkeys were highly experienced with the associative relationship between stimulus and reward; that is, the monkey used the stimulus as a predictor of the upcoming reward, as reflected by licking movements starting before reward delivery. We will refer to this situation as the reward-predicting stimulus condition. In the second condition, the same liquid reward was repeatedly delivered at irregular time intervals (5.5$8.5 \mathrm{~s}$ ) in the absence of any predictive stimulus. We will refer to this situation as the reward-only condition. The two conditions were presented as separate blocks of 30-40 trials each, the order of blocks being chosen randomly. In our previous work, we have demonstrated that the TAN response to a stimulus that predicts reward is paralleled by a lack of responsiveness to the reward itself (after Pavlovian conditioning), whereas most TANs remain responsive to unexpected deliveries of reward outside of any task (for review, see Apicella, 2007).

Our database consisted of 79 TANs recorded in the reward-predicting stimulus condition ( 26 from C, 25 from $\mathrm{P}$, and 28 from G) and 130 TANs recorded in the reward-only condition ( 23 from C, 10 from $\mathrm{G}$, and 97 from B). Eleven TANs were recorded in both conditions. The behavioral situations, recording methods, and TAN responses properties have been described previously (Ravel et al., 2003; Apicella et al., 2011; Deffains et al., 2010).

\section{Data analysis}

Analysis of short-latency responses of rat cholinergic interneurons to afferent stimulation. Peristimulus time histograms (PSTHs) were constructed from 50-200 consecutive stimulation trials with $2 \mathrm{~ms}$ bins and normalized to give firing probability (spikes per bin/trials). Cholinergic interneurons were considered to respond significantly at "short latency" if $\geq 20 \%$ of first spikes after the stimulus were fired in a response window of $1.5-20 \mathrm{~ms}$ and if, within this window, there was a histogram peak $>3$ SDs of the prestimulus ("baseline") firing probability (defined from -400 to $-100 \mathrm{~ms}$ before stimulation at $0 \mathrm{~ms}$ ). Neurons were considered to respond significantly at "long latency" if $20 \%$ of first spikes after the stimulus were fired in a response window of $20-50 \mathrm{~ms}$ and if there was a peak $>3$ SDs of the baseline in this window. The first spike in each stimulus trial, rather than the peak of the response in the PSTH, was therefore used to measure the response latency. The mean latencies of the first spikes in a given time window were compared using a Mann-Whitney U test or Wilcoxon signed-rank test as appropriate. The same short-latency analysis windows were also used for both paired-pulse and high-frequency train stimulation. For paired-pulse stimulation, latencies and firing probabilities across the first and second pulse were compared using Wilcoxon signed-rank tests as long as there were $\geq 5$ spikes in this window in response to both pulses. Because our high-frequency stimulation cycle was at $40 \mathrm{~Hz}$, we were able to perform the same analysis as for single stimulation within each $25 \mathrm{~ms}$ interval of the high-frequency stimulation pulse. Mean latency and firing probability were therefore calculated for each of the five pulses in the stimulation train. The cumulative sum of the firing probability across the five pulses and its slope was calculated to measure the magnitude of accumulated spiking through the stimulus train.

Characterization of multiphasic responses of rats and primate neurons using PSTHs. We designed a unified framework to characterize the multiphasic responses of both identified rat cholinergic interneurons to cortical/thalamic stimulation in anesthetized rats and of TANs to behaviorally relevant stimuli (reward-predicting cues or reward delivery with no cue) in monkeys. This involved a two-step approach in which we first calculated the overall temporal profile and established the class of response (any combination of initial excitation/pause/rebound) of each neuron based on its PSTH and then used this information to calculate parameters of the three phases for each trial.

First, a smoothed PSTH (50 ms bins with $90 \%$ overlap) was computed to define the response phases. After these PSTHs were normalized as a percentage of baseline firing, peaks and troughs were detected using a threshold of five contiguous bins over or under the 95\% confidence interval of the $400 \mathrm{~ms}$ prestimulus baseline firing in the PSTH (from -450 to $-50 \mathrm{~ms}$ for rats, from -500 to $-100 \mathrm{~ms}$ for monkeys). In both the rat and monkey datasets, the most elaborate population responses included early increases in firing rate (from here on defined as an "initial excitation"), followed by a decrease in or cessation of firing ("pause"), followed by a renewed increase in firing ("rebound"), as described previously (Schulz and Reynolds, 2013). Significant peaks and troughs in PSTHs were thus assigned to initial excitation, pause, and/or rebound phases based on their latencies compared with the population response and these previous investigations. For single-pulse stimulation in rats, the temporal boundaries of the response phases were as follows: initial excitation: 1-50 ms; pause: 50-400 ms; and rebound: 200-700 ms. For high-frequency train stimulation in rats, the boundaries were as follows: initial excitation: $1-150 \mathrm{~ms}$ (after the first pulse in the train); pause: 50-400 ms; and rebound: 200-700 ms. Therefore, for responses evoked by high-frequency electrical stimulation, the phase of initial excitation encompassed all spiking after the first pulse up until $25 \mathrm{~ms}$ after the last pulse. For primate TAN responses to behaviorally relevant stimuli, the boundaries were as follows: initial excitation: 10-200 ms (after the reward-predicting stimulus or reward delivery); pause: 75-350 ms; and rebound: $200-700 \mathrm{~ms}$. Note that the windows for the three phases are wide and overlapping to allow for variations in the temporal profile across neurons and jitter in the response latencies. Significant responses were defined as such when they were detected at any time within these boundaries. This is particularly relevant for the primate data in which the latencies for each phase were longer for the reward-only condition. Based on the combination of peaks and troughs displayed, neurons responses were assigned to one of eight categories: Unclassified, Initial excitation only, Pause only, Rebound only, Initial excitation/Pause, Initial excitation/ Pause/Rebound, Initial excitation/Rebound, and Pause/Rebound. The smoothed PSTHs were also used to define the start and end of each response phase. Every PSTH was extensively scrutinized to verify that these boundaries and other criteria led to satisfactory response classification.

Trial-by-trial analysis of multiphasic responses of neurons in rats and primates. Having defined the response class and the timing of each PSTH response phase for each neuron in rat and monkey, we used these data to analyze the relationship between the different response phases on a trialby-trial basis. The PSTH response classification allowed us to establish that the majority of rat and monkey neurons displayed a pause (see Results). Therefore, for each neuron that had a pause in the PSTH, we 
used the pause onset, defined by the first bin in the PSTH that fell below the $95 \%$ confidence interval, as a reference point to calculate a value for the number of spikes in the preceding initial excitation, the duration of the pause itself, and the change in firing rate during the subsequent rebound phase for every stimulus trial.

Initial excitation. This initial excitation phase for each trial was defined as the number of spikes fired between the "trigger" (the first electrical stimulation in rat data or the visual cue or reward delivery in primate data) and $50 \mathrm{~ms}$ after the start of the pause onset (to allow for single trials in which the initial excitation lasted longer that the PSTH pause onset) as defined by the PSTH. Trials could then be separated based on how many spikes were fired in this initial excitation phase and PSTHs recalculated to include only the trials with a given number of spikes in this window. For group statistics, the number of spikes in each trial was averaged for each individual rat cholinergic interneuron or primate TAN.

Pause. The pause phase for each trial was defined as the interspike interval between the last spike of the initial excitation phase and the next spike. For trials in which no spikes were fired in the initial excitation window (i.e., no activity up to $50 \mathrm{~ms}$ after the start of the PSTH pause), the start of the pause for that trial was the start of the PSTH pause (i.e., the first bin in the PSTH to be significantly below the baseline). The individual pause values for each trial were normalized by the mean interspike interval of the baseline period for the entire set of trials (Ding et al., 2010). For group statistics, the mean pause for each neuron was the mean pause/ trial divided by the mean interspike interval during baseline firing. Therefore, a value of $>1$ indicates that the pause interval is greater than the mean interspike interval of the baseline.

Rebound. The rebound phase for each trial was defined as the firing rate in the $500 \mathrm{~ms}$ after the pause interval for that trial (including the spike that defined the pause offset) normalized by the mean firing rate of the baseline period. Therefore, the "rebound rate" was the percentage change in the firing rate of the $500 \mathrm{~ms}$ after the pause compared with the baseline rate.

This trial-by-trial approach had several advantages compared with analyzing responses based on the PSTH. First, the calculation of these values was not directly dependent on the binning of data for the PSTH. Second, in the cases for which there was no initial excitation or rebound peak in the PSTH, it was still possible to calculate the values for these response phases and to detect single trials that did have increases in spiking during these phases. Third, by defining values for each response phase in each trial, we could isolate and sort trials based on specific characteristics (e.g., one, two, or three spikes in the initial excitation phase/sorting trials by pause length). Finally, using these methods enabled us to focus our analyses on changes in firing rate within a trial, not consistency in latency with respect to the stimulus across trials. Such changes are less dependent on binning and likely to be more relevant to the online computation performed by the neuron. In summary, our analyses enabled us to investigate, within the same analytical framework, the multiphasic responses of both rat cholinergic interneurons after electrical stimulation and monkey TANs after rewarding stimuli.

\section{Results}

\section{Cortical and thalamic inputs to individual cholinergic interneurons in the rat}

Although cholinergic interneurons only make up a small proportion of striatal neurons, it is widely accepted that these interneurons play an important role in reward-related behavior through the modulatory actions of the acetylcholine that they release within the striatum. In the first part of this study, cholinergic interneurons were recorded in the striatum of anesthetized rats. Online identification of putative cholinergic interneurons was facilitated by previous work in urethane-anesthetized rats (Sharott et al., 2012) showing that, regardless of brain state, cholinergic interneurons fire spontaneously at similar rates $(3-6 \mathrm{~Hz}$; Fig. $1 A$ ). After the recording of spontaneous activity, the same interneurons were then recorded during the delivery (in random order) of a variety of electrical stimuli to the cortex (ipsilateral motor cortex) and/or thalamus (nominally targeted to ipsilateral parafascicular nucleus). An individual cholinergic interneuron could fire at short latencies ( $<20 \mathrm{~ms})$ in response to single-pulse stimulation of both cortical and thalamic sites (Fig. $1 B, C$ ). The interneuron shown in Figure 1 responded to cortical stimulation with an average delay to first spike of $9.1 \mathrm{~ms}$ (Fig. 1B) and to thalamic stimulation with an average delay of $12.7 \mathrm{~ms}$ (Fig. 1C). These and similar short-latency excitation responses were assumed to reflect activation of monosynaptic inputs. Note, however, that the responses of interneurons to single-pulse afferent stimulation were often multiphasic. Therefore, short-latency excitations were often followed by (in order) a cessation of firing and a subsequent rebound increase in firing (Fig. $1 B, C$ ). After recording and electrical stimulation, interneurons were juxtacellularly labeled with NB (Fig. 1D) and subsequently tested for the expression of ChAT immunoreactivity (Fig. 1E). All rat neurons included in this study were confirmed to express ChAT and were thus unequivocally identified as cholinergic interneurons.

The synaptic innervation of three physiologically and neurochemically characterized cholinergic interneurons was examined at the EM level. The somata and dendrites of these cholinergic interneurons were first digitally reconstructed in 3D (Fig. $1 F$ ). Alternate tissue sections were incubated in antibodies against either vesicular glutamate transporter 1 or 2 (VGluT1 or VGluT2) to quantify inputs from cortex and thalamus, respectively (Doig et al., 2010; Henny et al., 2012; Henny et al., 2013) and then processed for EM. One identified cholinergic interneuron (\#AJS044) that exhibited robust responses to cortical and thalamic stimulation in vivo (the same neuron as shown throughout Fig. 1) was shown to form synapses with axons originating in both the cortex (VGluT1 positive; Fig. $1 G$ ) and the thalamus (VGluT2 positive; Fig. $1 H$ ). This establishes the precedent that an individual cholinergic interneuron can form synapses with axon terminals arising from both the cortex and thalamus. Further details of the synaptic innervation of cholinergic interneurons are discussed below.

\section{Synaptic innervation of cholinergic interneurons}

A detailed anatomical examination of the synaptic inputs to three identified cholinergic interneurons was performed using EM. The NB-filled neurons were first reconstructed in 3D before reembedding and resectioning for EM (Henny et al., 2012; Henny et al., 2013). In agreement with previous studies, cholinergic interneurons had distinctly large cell bodies, and between three and six primary dendrites that extend in a radial pattern from the soma for up to $\sim 700 \mu \mathrm{m}$ (e.g., Fig. 1F; Wilson et al., 1990; Inokawa et al., 2010; Sharott et al., 2012). The average total dendritic length for the three neurons reconstructed was $9411 \mu \mathrm{m}( \pm 511)$.

After reconstruction, fragments of dendrites from all parts of the dendritic arbor were sectioned for analysis in the electron microscope. For each neuron, serial sections of 20-24 dendritic fragments were examined in tissue labeled for either VGluT1 or VGluT2 (Table 1). Dendritic fragments were examined in serial sections in tissue that exhibited staining for VGluT1 or VGluT2. On average, 42 sections were analyzed for each of the dendritic fragments, giving an overall average of 892 sections per neuron (Table 1). All terminals forming synapses with the dendrites were noted (Fig. 2, Table 1) and categorized as follows: (1) DABnegative (unlabeled) terminals forming asymmetric (Gray's type I) synapses (Fig. 2A-C, Table 1); (2) DAB-negative (unlabeled) terminals forming symmetric (Gray's type II) synapses (Fig. 2D, Table 1); and (3) DAB-positive (i.e., VGluT1-or VGluT2immunopositive) terminals forming asymmetric synapses (Figs. $1 E, F, 2 B$, Table 1). 

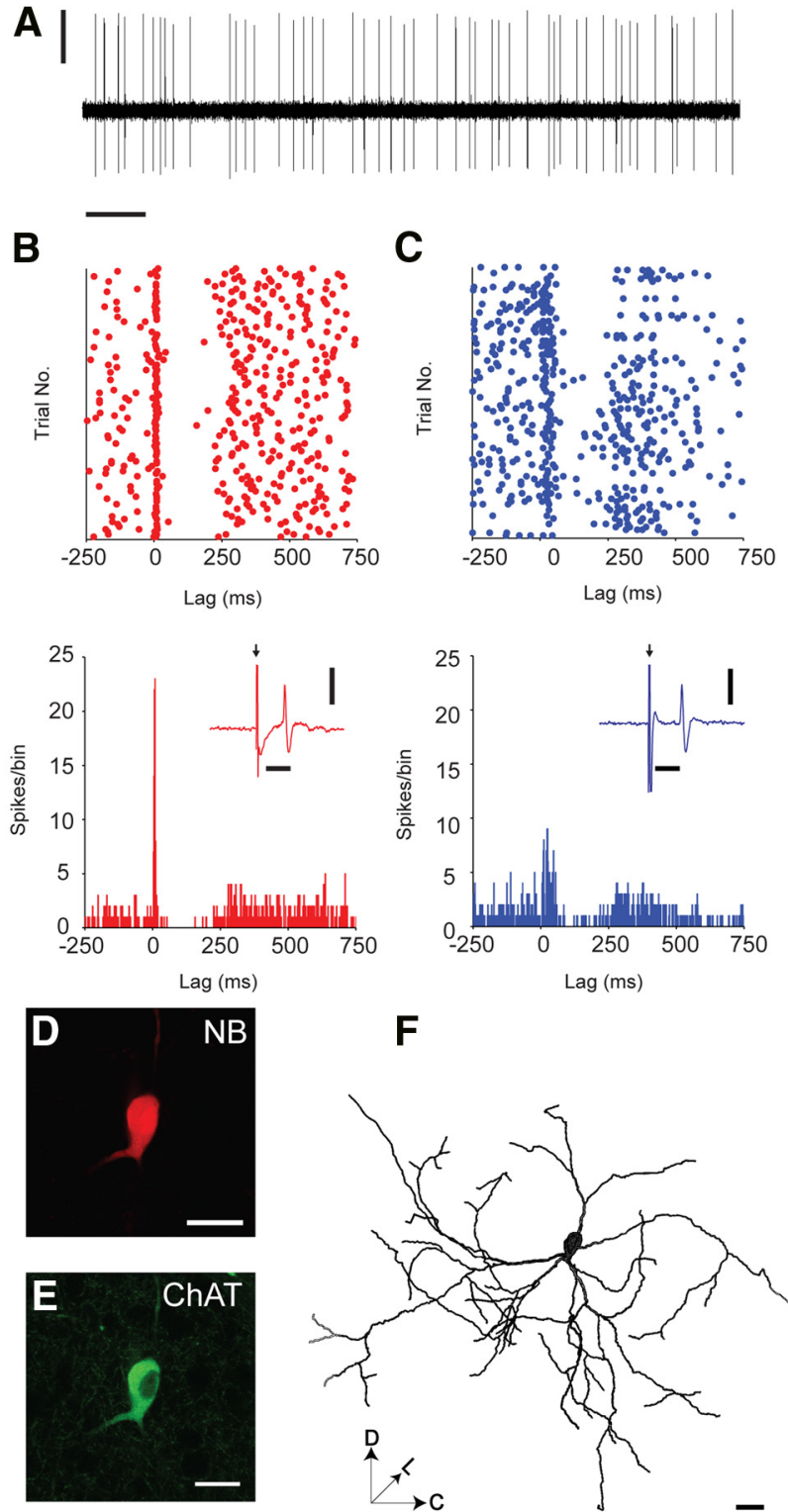

G

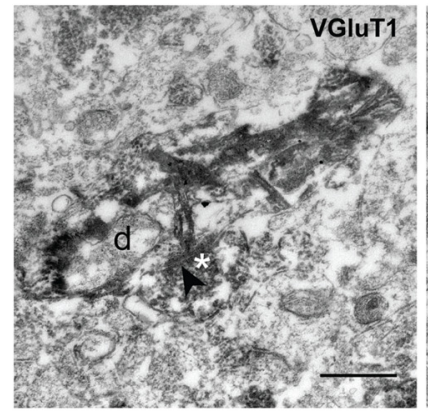

$\mathbf{F}$

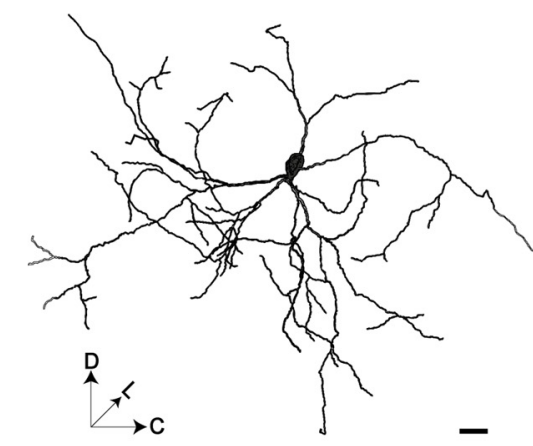

H

Figure 1. Individual identified cholinergic interneurons can respond to cortical and thalamic stimulation and receive synaptic input from the cortex and the thalamus. $A$, Recording of the spontaneous spike firing of an individual cholinergic interneuron (\#AJS044) in an anesthetized rat; vertical scale bars, $1 \mathrm{mV}$; horizontal scale bar, 1 s. B. Raster plot (top) and PSTH (bottom) showing the response of interneuron in $\boldsymbol{A}$ to single-pulse stimulation of ipsilateral motor cortex. A single-trial example of an evoked spike waveform after cortical stimulation (arrow) is inset; horizontal scale bar, $5 \mathrm{~ms}$; vertical scale bar, $1 \mathrm{mV}$. C, Raster plot (top) and corresponding PSTH (bottom) showing the response of the same interneuron to single-pulse stimulation (arrow) of the ipsilateral thalamus (targeted to the parafascicular nucleus). An example of an evoked spike waveform after thalamic stimulation is inset; horizontal scale bar, $5 \mathrm{~ms}$; vertical scale bar, $1 \mathrm{mV}$.

Table 1. EM analysis of the synaptic innervation of cholinergic interneuron dendrites

\begin{tabular}{lrrrrr}
\hline & \multicolumn{3}{l}{ Neurons } & & \\
\cline { 2 - 4 } Properties examined & NJX09 & AJS044 & NJX014 & Average \\
\hline Total number of ultrathin sections examined (50 nm) & 849 & 1039 & 789 & 892.33 \\
Total number of dendritic fragments analyzed & 20 & 24 & 20 & 21.33 \\
$\quad$ In VGluT1-labeled tissue & 11 & 14 & 11 & 12.00 \\
$\quad$ In VGluT2-labeled tissue & 9 & 10 & 9 & 9.33 \\
Total number of unlabeled (DAB-negative) & 23 & 32 & 46 & 33.67 \\
$\quad$ asymmetric synapses & 11 & 22 & 27 & 20.00 \\
$\quad$ In VGluT1-labeled tissue & 12 & 10 & 19 & 13.67 \\
In VGluT2-labeled tissue & 0 & 2 & 0 & 0.67 \\
Total number of VGluT1-positive synapses & 6 & 5 & 3 & 4.67 \\
Total number of VGluT2-positive synapses & 61 & 62 & 64 & 62.33 \\
Total number of (unlabeled) symmetric synapses & 61 & & & \\
\hline
\end{tabular}

For each neuron examined, several properties were analyzed over a number of sections in either VGluT1- or VGluT2labeled tissue.

Overall, $62 \%$ of the terminals in contact with the dendrites of cholinergic interneurons formed symmetric synapses (Fig. 2E) and $38 \%$ formed asymmetric synapses $(p=0.05$; Mann-Whitney $\mathrm{U}$ test, two-tailed, Fig. $2 E$ ). Of the terminals that formed asymmetric synapses, $1.7 \%$ were positive for VGluT1 and $13 \%$ for VGluT2 (Fig. 2E, inset). The remainder of terminals forming asymmetric synapses were DAB negative in tissue labeled for either VGluT1 $(49.8 \pm 3.4 \%)$ or VGluT2 (35.3 $\pm 2.8 \%$; Fig. $2 E$, inset). Therefore, the predominant type of synapse formed with the dendrites of cholinergic interneurons was symmetric and formed by unlabeled terminals.

We then examined the distribution of synapses formed with the cholinergic neurons in different parts of the dendritic arbor (Fig. 2F, Table 2). Dendrites were defined as "proximal" to the soma (i.e., within the first $20 \%$ of the total length from the soma of the longest dendrite; Henny et al., 2012; Henny et al., 2013), "distal" (21-80\%); or "most distal" (the farthest $20 \%$ of the distance of dendrites from the soma; Table 2). The number and type of terminals forming synapses was examined within each compartment (Table 2). The number of terminals forming asymmetric or symmetric synapses was normalized based on the length of dendrite within each compartment for each dendritic fragment (Fig. 2F). Of the two terminals positive for VGluT1, one was on a proximal dendrite and the other on a distal dendrite (Table 2). Synapses formed by terminals positive for VGluT2 were found in all dendritic compartments (Table 2).

Overall, the average density of terminals forming asymmetric synapses per $10 \mu \mathrm{m}$ of dendrite was $9.9 \pm 1.1$ compared with $14.4 \pm 1.2$ symmetric synapses; therefore, over the entire dendritic tree, the density of symmetric synapses was significantly greater than the density of asymmetric synapses ( $p=0.0038$; MannWhitney $\mathrm{U}$ test, two-tailed). This ratio of symmetric to asymmetric synapses is in agreement with previous data (Sizemore et al., 2010). Indeed, in all compartments, there were more terminals forming

$\leftarrow$

Note the short-latency excitations ( $<20 \mathrm{~ms}$ ) and multiphasic responses evoked by cortical and thalamic stimuli. $\boldsymbol{D}, \boldsymbol{E}$, After recording, the same interneuron was juxtacellularly labeled with NB and tested positive for immunoreactivity against ChAT, thus confirming its cholinergic identity. Scale bars, $25 \mu \mathrm{m}$. $\boldsymbol{F}$, Somata and dendrites of the identified cholinergic neuron digitally reconstructed in 3D. Scale bar, $25 \mu \mathrm{m}$. $\mathbf{G}, \boldsymbol{H}$, The same interneuron was then examined using electron microscopy. In $\mathbf{G}$, a dendrite (d) is shown forming an asymmetric synapse (arrowhead) with an axon terminal (white asterisk) that is positive for VGluT1, a marker of cortical terminals. Note the crystalline deposits in the dendrite formed by the TMB. Scale bar, $0.25 \mu \mathrm{m}$. In $\boldsymbol{H}$, another dendrite (d) is shown forming an asymmetric synapse (arrowhead) with an axon terminal (white asterisk) that is positive for VGluT2, a marker of thalamic terminals. 

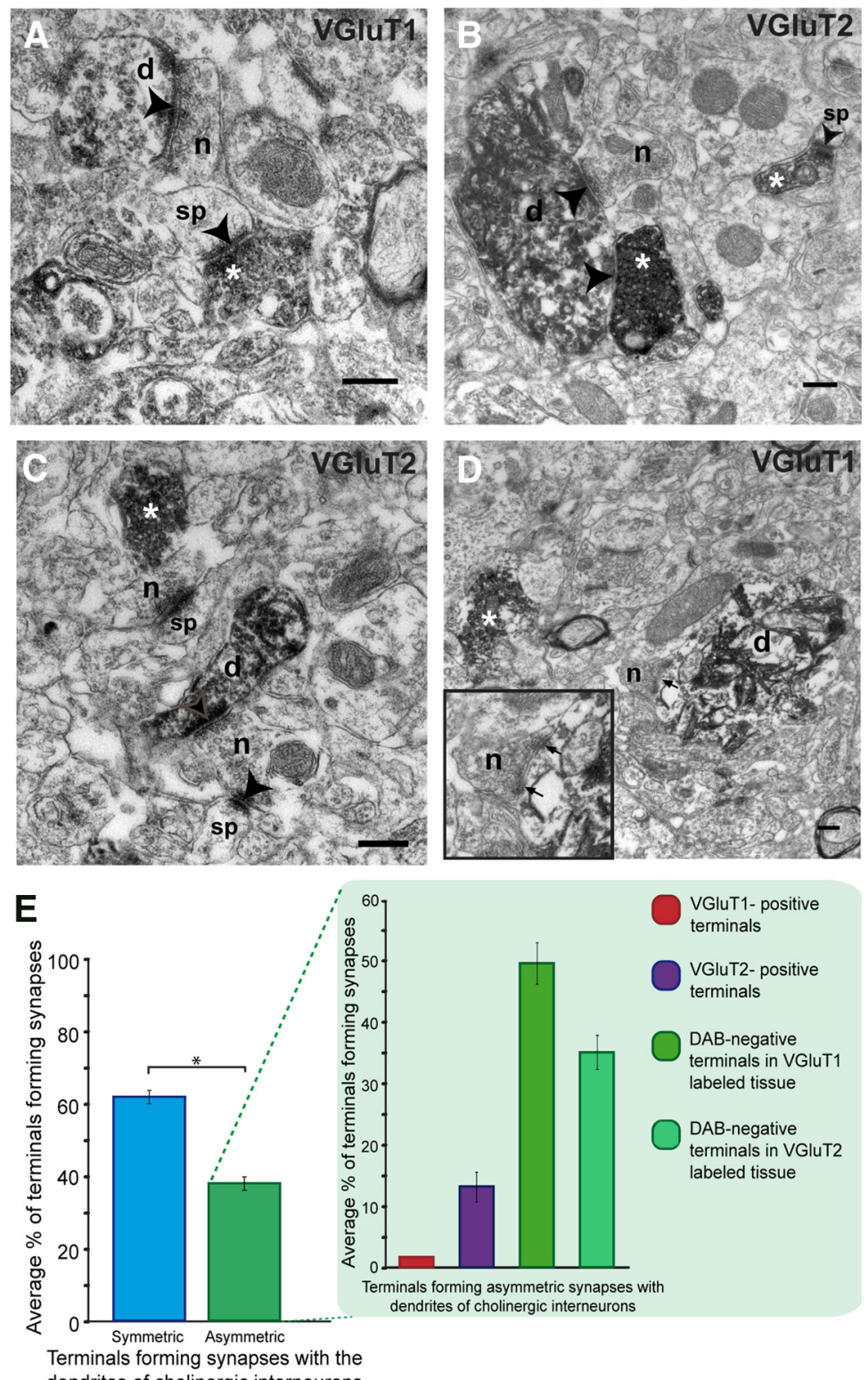
dendrites of cholinergic interneurons
$\mathbf{F}$

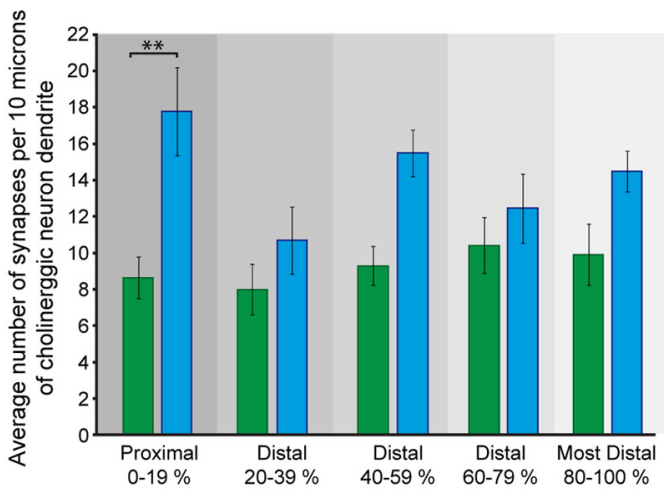

Distance from the soma
G

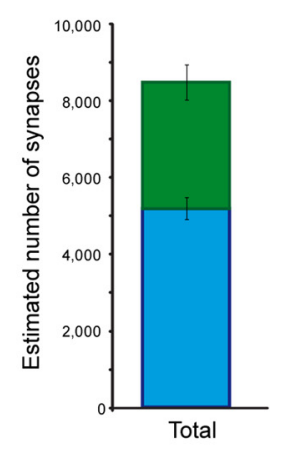

Figure 2. Terminals forming synapses with the dendrites of cholinergic interneurons. $\boldsymbol{A}$, Example of a dendrite of a cholinergic interneuron (d) forming an asymmetric synapse (arrowhead) with a terminal negative for VGluT1 (n). Note that there is a terminal positive for VGluT1 (DAB product; white asterisk), forming an asymmetric synapse (arrowhead) with a MSN spine (sp). B, Cholinergic interneuron dendrite (d) forms two asymmetric synapses (arrowheads) with a VGluT2 positive terminal (asterisk) and terminal negative for VGluT2 ( $n$ ). Note that within the same frame there is another positive terminal (asterisk) forming a synapse symmetric than asymmetric synapses; however, this was most prominent proximal to the soma, with asymmetric synapses forming $8.6 \pm 1.1$ synapses per $10 \mu \mathrm{m}$ of dendrite and symmetric synapses forming $17.8 \pm 2.4$ synapses, and indeed this was significant in this proximal compartment $(p=0.0081$, Mann-Whitney U test, two-tailed; Fig. $2 F$ ). In all other compartments, the density of symmetric synapses was not significantly greater than the density of asymmetric synapses $(p>0.05$, Mann-Whitney $\mathrm{U}$ test, two-tailed; Fig. $2 F)$.

Due to the way in which we sampled the synapses, we were able to extrapolate our data and estimate the total numbers of synapses formed with an individual cholinergic interneuron by the various different terminals. Our data suggest that an individual cholinergic interneuron has an average of $8450 \pm 694$ afferent synapses in total (Fig. 2G), of which the majority are symmetric (an average of $5166 \pm 285$; Fig. $2 G$ ) and the remainder are asymmetric (an average of $2859 \pm 458$; Fig. $2 G$ ). We estimate that an individual cholinergic interneuron forms an average of $752 \pm 62$ synapses with VGluT2-positive terminals. The data also indicate that neuron AJS044 would form a total of 294 synapses with VGluT1-positive terminals. It is clear from these data that cholinergic interneurons form more synapses with thalamic than cortical terminals, which is in agreement with previous data (Lapper and Bolam, 1992).

\section{Short-latency responses of rat} cholinergic interneurons to cortical and thalamic stimulation

The finding that an individual cholinergic interneuron can receive synaptic input

$\leftarrow$

with an MSN spine (sp). C, A dendrite (d) forms an asymmetric synapse (arrowhead) with a terminal negative for VGluT2 (n). Note that the same terminal is also forming a synapse with a spine of an MSN (sp) and that within the same frame there is a VGluT2 positive terminal (asterisk). There is also a negative terminal ( $n$ ) forming a synapse with a spine (sp). $\boldsymbol{D}$, The dendrite of a cholinergic interneuron (d) forms a symmetric synapse (small arrows) with an unlabeled terminal (n), see inset. Note that within the frame there is a terminal positive for VGluT1. Scale bars, $0.25 \mu \mathrm{m}$. $\boldsymbol{E}$, Percentages of terminals forming symmetric (blue) or asymmetric (green) synapses with the dendrites examined. of the terminals that formed asymmetric synapses (green), some were positive for VGluT1 (red) or VGluT2 (violet). The remaining terminals were DAB negative in tissue labeled for either VGluT1 or VGluT2 (inset). $\boldsymbol{F}$, Average number of asymmetric (green) and symmetric (blue) synapses normalized for every $10 \mu \mathrm{m}$ of dendrite, within each compartment. G, Estimations of the total number of symmetric (blue) and asymmetric (green) synapses formed with the dendrites of cholinergic interneurons based on data collected. 
Table 2. Distribution of synapses over the dendritic arbor of cholinergic interneurons

\begin{tabular}{|c|c|c|c|}
\hline Properties examined & Proximal $(0-19 \%)$ & Distal $(20-79 \%)$ & Most distal $(80-100 \%)$ \\
\hline Number of dendritic fragments analyzed & 17 & 38 & 9 \\
\hline Average distance from soma ( \pm SEM) $(\mu \mathrm{m})$ & $59.96( \pm 12.19)$ & $276.53( \pm 34.22)$ & $558.22( \pm 17.00)$ \\
\hline Average distance from soma as a percentage of total distance ( \pm SEM) & $9.31( \pm 1.90)$ & $43.64( \pm 5.40)$ & $85.48( \pm 2.17)$ \\
\hline Ultrathin sections analyzed (total) & 730 & 1568 & 379 \\
\hline Average number of ultrathin sections analyzed in serial section ( \pm SEM) & $42.94( \pm 7.02)$ & $41.26( \pm 6.26)$ & $42.11( \pm 10.13)$ \\
\hline Total number of terminals forming asymmetric synapses & 24 & 58 & 19 \\
\hline In VGluT1-labeled tissue & 14 & 40 & 6 \\
\hline In VGlut2-labeled tissue & 10 & 18 & 13 \\
\hline Total number of VGlut1-positive terminals forming synapses & 1 & 1 & 0 \\
\hline Total number of VGlut2-positive terminals forming synapses & 2 & 10 & 2 \\
\hline Total number of terminals forming symmetric synapses & 55 & 103 & 29 \\
\hline
\end{tabular}

The distribution of terminals forming synapses on dendrites at varying distances from the soma was analyzed for the three neurons examined.

from both the cortex and the thalamus concurred with the responses of these interneurons to electrical stimuli delivered to these two regions in vivo. Thirty-two identified cholinergic interneurons were included in this study, 18 of which were recorded with cortical stimulation only; the remaining 14 were recorded during both cortical and thalamic stimulation (delivered independently and in a random order). As a first step, the responses of identified cholinergic interneurons to brief, singlepulse electrical stimulation of the ipsilateral motor cortex $(n=$ 32 ) and Pfn $(n=14)$ were examined (Fig. 3$)$. Studies in vitro have reported that excitation of either cortical or thalamic axons with single electrical pulses does not elicit spiking responses from cholinergic interneurons (Oswald et al., 2009; Ding et al., 2010; Schulz et al., 2011). In contrast, we found that the firing of many cholinergic interneurons was transiently increased at "short latencies" of 1.5-20 ms in response to single-pulse stimulation of the cortex (50\% of interneurons tested) and thalamus (64\%) in vivo (Fig. $3 A-F$ ). Short-latency decreases in interneuron firing were not observed in response to afferent stimulation. Evoked spiking at these short latencies is thought to be indicative of monosynaptic inputs (Mallet et al., 2005; Sharott et al., 2012) and likely requires many corticostriatal or thalamostriatal fibers to be recruited by the stimulation. In vitro preparations, which entail the loss of at least some connections, may thus not allow for the recruitment of enough afferent neurons/axons with a singlepulse stimulus to elicit interneuron spiking.

The mean latencies of first spikes in this $1.5-20 \mathrm{~ms}$ window did not significantly differ between responses to cortical (10.2 \pm $0.49 \mathrm{~ms})$ and thalamic $(10.7 \pm 0.57 \mathrm{~ms})$ stimulation (MannWhitney, $p>0.05$; Fig. $3 G$ ). This was also the case for individual neurons that responded to both cortical and thalamic stimulation (Wilcoxon signed-rank test, $p>0.05$; Fig. $3 H$ ). The majority of cholinergic interneurons ( 10 of 14 tested) also responded to thalamic, but not cortical (Fig. $3 B, C$, inset), stimulation at a longer latency of 20-50 ms (mean latency: $33.6 \pm 0.58 \mathrm{~ms}$; Fig. $3 E, F$, inset). This later component could be due to a recruitment of polysynaptic circuits that ultimately impinge on striatum, such as cortical fibers that might be activated by ITN stimulation (Llinas et al., 2002). In summary, these group data show that cholinergic interneurons can respond with a short-latency increase in firing, indicative of a monosynaptic drive.

In vivo, striatal projection neurons and parvalbuminexpressing $(\mathrm{PV}+)$ interneurons show distinct and opposite responses to paired-pulse stimulation (100 ms interpulse interval) of the ipsilateral frontal cortex (Mallet et al., 2005). We next examined the short-latency responses of cholinergic interneurons to paired-pulse stimulation (100 ms interval) of either cortex or thalamus (Fig. $4 A, B$ ). For this protocol, we tried to apply a stimulation current that achieved a spike response to the first pulse around the rheobase (i.e., the current amplitude that evokes a spike after $50 \%$ of first pulses). With paired-pulse stimulation of cortex, cholinergic interneurons had a significantly lower probability of short-latency firing to the second pulse (Fig. 4C), as has been described previously for PV + interneurons (Mallet et al., 2005). Note that the short-latency response to the second pulse decreased across the whole range of initial firing probabilities and was not therefore a phenomenon limited to an initial high firing probability (Fig. $4 C$ ). In contrast, there was no change in firing probability across the first and second pulses of thalamic stimulation (Fig. 4D). Although the firing probability of the longer-latency responses was greater after the second pulse for some neurons (Fig. 4B), this was not significant across the population. Therefore, in a similar manner to PV + interneurons, cholinergic interneurons respond preferentially to the first pulse in a paired-pulse stimulation protocol for cortical stimulation. This relationship was not observed for thalamic stimulation, suggesting that: (1) electrical stimuli delivered to cortical and thalamic sites recruited distinct sets of inputs and (2) that there is a fundamental difference in the integration of the two excitatory afferents by cholinergic interneurons.

Previous studies, both in vitro and in vivo, have suggested that burst-like excitatory inputs to cholinergic interneurons evoke responses that mimic the timing of these neurons to motivationally salient stimuli in behaving animals (Nanda et al., 2009; Oswald et al., 2009; Ding et al., 2010). Therefore, we next examined the responses of cholinergic interneurons to short trains of highfrequency stimuli ( 5 pulses at $40 \mathrm{~Hz}$ ) delivered independently to cortex and thalamus. Short-latency interneuron responses could be readily discriminated after one or more pulses of the stimulation trains (Fig. 5A,B). Cortical stimulation led to more spikes being fired in response to the first pulse, followed by a gradual decrease in short-latency firing probability throughout the stimulus train (Fig. 5C), which is consistent with interneuron responses to the paired-pulse stimulation at $10 \mathrm{~Hz}$ (Fig. 4). Conversely, the short-latency firing probability increased through the stimulus train in response to thalamic train stimulation, with the maximum response after the third pulse of the stimulus (Fig. 5D). The difference in firing probability for the first cortical stimulation pulse and the mean of the following stimuli (pulses 2-5) narrowly missed significance $(p=0.05$, Wilcoxon signed-rank test; Fig. $5 E$ ), but the difference was highly significant when the response was normalized to give the number of spikes as a percentage of spikes fired across all pulses, thus taking into account baseline firing rate (Fig. $5 F$ ). The firing probability for the first thalamic stimulation was significantly lower than the mean of the following stimuli (pulses 2-5) for both raw and normalized mea- 


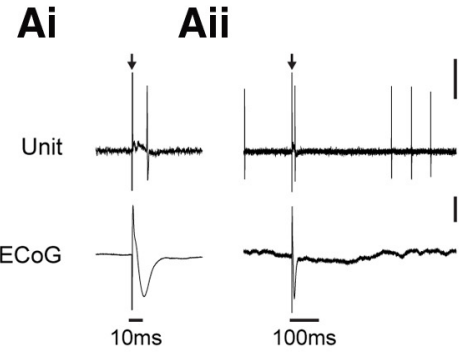

B

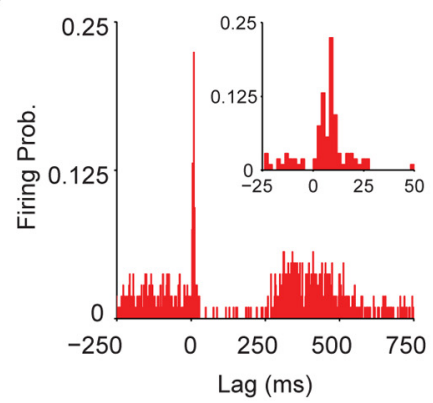

C
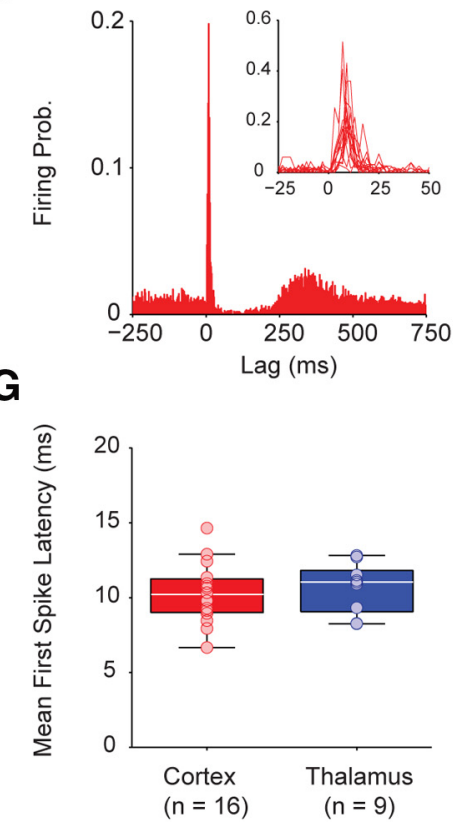

Di
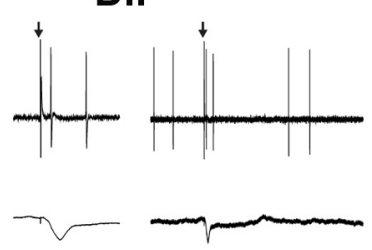

$\overline{10 \mathrm{~ms}} \quad \overline{100 \mathrm{~ms}}$

E

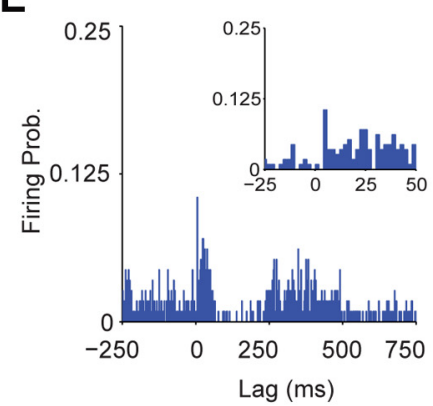

$\mathbf{F}$

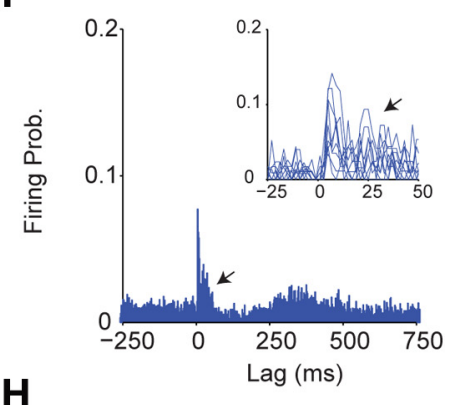

H

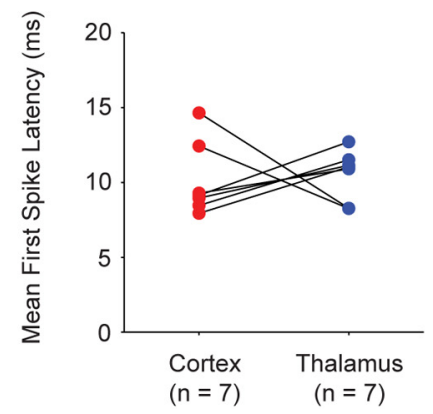

Figure 3. Short-latency responses of cholinergic interneurons to cortical and thalamic stimulation. Ai, Unit-activity recording (single representative sweep of stimulation artifacts and evoked responses, arrow indicates stimulation onset) of a cholinergic interneuron that fired at short latency ( $\sim 10 \mathrm{~ms})$ in response to single-pulse electrical stimulation of the cortex. The coincident ECOG is shown below; note evoked potential. Aii, Expanded view of the same response showing that, after the short-latency spike, there is a cessation of firing $(\sim 250 \mathrm{~ms})$ followed by a period of renewed spiking. $\boldsymbol{B}$, PSTHs ( $2 \mathrm{~ms}$ bins) of the same interneuron's response to cortical stimulation (single pulses delivered at $0 \mathrm{~ms}$, with artifact removed). Inset, Response during the first $50 \mathrm{~ms}$ after stimulation. C, Mean PSTH of all cholinergic interneurons that showed a significant response to cortical stimulation $(n=16)$. Inset, Responses of each interneuron (thin lines) during the first $50 \mathrm{~ms}$ after stimulation. Di, The same interneuron (as in $\boldsymbol{A}$ ) also fired at short latency $(\sim 10 \mathrm{~ms}$ ) in response to single-pulse electrical stimulation of the thalamus. A second spike follows after $\sim 40 \mathrm{~ms}$. Dii, Expanded view highlighting a later pause in firing that is followed by renewed spiking. $\boldsymbol{E}$, PSTHs (2 ms bins) of the same interneuron's response to thalamic stimulation (single pulses delivered at $0 \mathrm{~ms}$ ). Inset, Response during the first $50 \mathrm{~ms}$ after stimulation. $\boldsymbol{F}$, Mean PSTH for all interneurons that responded significantly to thalamic stimulation $(n=9)$. Note the evoked increase in firing between 25 and 50 ms (arrowhead) that is not present after cortical stimulation (see $\boldsymbol{C}$ ). G, Latencies of the first spikes evoked at short latency (lag of 1.5-20 ms) of all interneurons significantly responding to either cortical or thalamic stimulation. Box plots show the medians (white line), the interquartile ranges (box), and extremes of the range (whiskers, within $99 \%$ of the distribution). Dots show the mean latency of each individual interneuron. $\boldsymbol{H}$, Latencies of the first spikes evoked at short latency in all interneurons significantly responding to both cortical and thalamic stimulation. Lines join data from individual interneurons.

sures (Fig. $5 E, F$ ). Because of this facilitation of firing through the thalamic stimulation train, and despite having a lower probability of firing to the first pulse, thalamic $40 \mathrm{~Hz}$ stimulation led to a significantly greater number of evoked spikes for the whole stimulation period $(0-125 \mathrm{~s})$ compared with firing evoked by cortical stimulation ( $p<0.05$, MannWhitney $U$ test). The cumulative sum of the spikes at each pulse demonstrated the greater number of spikes at the end of the thalamic stimulus train (Fig. 5G), so the slope of this line was significantly steeper for thalamic than cortical stimulation (Fig. $5 H$ ). It is noteworthy that not only did the firing probability increase in response to thalamic stimulation, but at its peak (i.e., the third pulse), it reached the same level (or higher) as that of the first cortical stimulus pulse (Fig. $5 E$ ). In summary, the probability of cholinergic interneurons firing in response to the stimulation of excitatory afferents was therefore highly dependent on history (the preceding inputs) and the source of input activity (cortical vs thalamic).

The stimulation currents applied to cortex and thalamus were not significantly different in the single, paired-pulse, or high-frequency stimulation conditions (Mann-Whitney, $p>0.05$ ). Differences in the responses of cholinergic interneurons to cortical and thalamic stimulation were therefore not due to a systematic difference in the strength of stimulation.

\section{Multiphasic responses of cholinergic interneurons to cortical and thalamic stimulation}

Our anatomical results provided a structural substrate for the short-latency increases in firing of cholinergic interneurons in response to cortical and thalamic stimulation. In behaving animals, however, the majority of studies on TANs (i.e., presumed cholinergic interneurons) have focused on the pause in their firing and subsequent rebound firing in response to salient stimuli (Aosaki et al., 1994; Morris et al., 2004). Most of the identified cholinergic interneurons we recorded in rats also displayed multiphasic responses after excitatory afferent stimulation (Figs. 1, 3, $4,5)$. As described previously, the three most prominent phases of the response were an initial increase in firing occurring shortly after stimulation, followed by a decrease or cessation of firing, and then a renewed or rebound increase in firing rate compared with baseline. To facilitate the comparison of data from rat and monkeys (see below), we term these three phases 
"initial excitation," "pause," and "rebound," respectively. Note that the initial excitation is defined here as the number of spikes fired directly after stimulation (and before the pause), not just those in the short-latency window $(<20 \mathrm{~ms})$ that we used above to infer putative monosynaptic connectivity. In some studies (Ding et al., 2010; Schulz and Reynolds, 2013), "burst" has been used to describe the initial excitation, but we refrain from using this term because single trials were generally not accompanied by $\geq 2$ spikes in this phase. For each identified cholinergic interneuron, we first defined which of these phases occurred in the PSTH for singlepulse and/or high-frequency train stimulation of the cortex and/or thalamus and then classified them by response type accordingly (Fig. 6). The majority of interneurons displayed all three phases in response to both single-pulse and $40 \mathrm{~Hz}$ stimulation of cortex (Fig. 6A,B), although approximately one-third of interneurons had the pause and rebound response phases after single-pulse stimulation (Fig. 6A). The majority of cholinergic interneuron responses to thalamic stimulation $(79 \%)$ also had initial excitation/pause/rebound phases, but this changed markedly during high-frequency stimulation, in which only $22 \%$ of responses displayed the rebound phase (Fig. $6 C, D)$. The pause was, therefore, almost ubiquitous after single-pulse or high-frequency train stimulation of excitatory afferents. More often than not, pauses were preceded by an initial excitation. In contrast, rebound firing was uncommon after high-frequency thalamic stimulation, which also resulted in the highest amount of initial excitation.

The prevalence of pause and rebound phases of interneuron responses to electrical stimulation of thalamus and cortex suggests that, through interaction with intrinsic cellular and/or circuit mechanisms, monosynaptic excitation of cholinergic interneurons may also lead to multiphasic responses. We used the variation in stimulation-evoked spiking across all of our stimulation protocols to test the hypothesis that the temporal profile of the multiphasic response was dependent on the level of initial excitation. To compare our findings in rat with those in behaving monkeys, in which it was not possible to discriminate between cortical and thalamic inputs, we looked for relationships with excitation per se (i.e., those that were consistent across stimulation site and protocol). Previous investigations have demonstrated that the length of the pause phase of rodent interneurons is dependent on the amount of spiking or depolarization after stimulation of cortical and thalamic axons (Oswald et al., 2009; Ding et al., 2010). We investigated whether this phenomenon (as reflected in spiking) could also be seen in our in vivo rat data and whether the variance in the initial excitation and pause phases could also predict the magnitude of the rebound phase (Fig. 7).

Raster plots of individual interneurons responding to singlepulse cortical and thalamic stimulation, in which the trials have been sorted by pause duration, reveal that in trials with relatively short pauses, the pause was often preceded by one or no spikes in

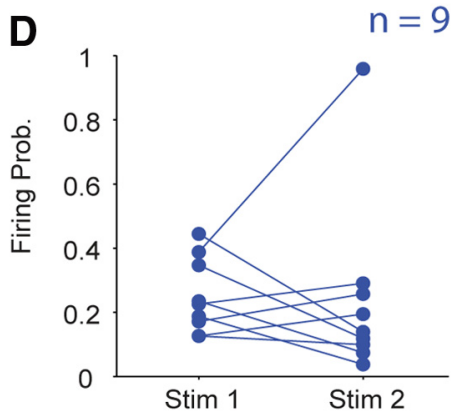

B
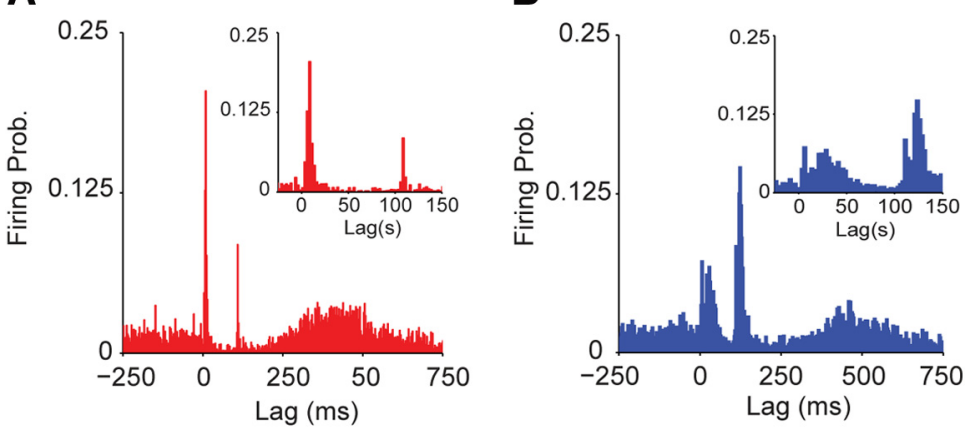

.

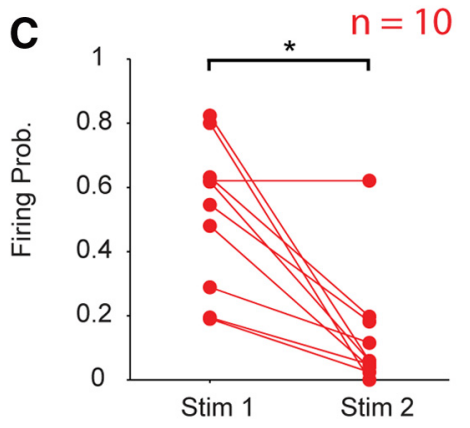

Figure 4. Paired-pulse stimulation of cortex, but not thalamus, leads to a decrease in the evoked firing probability of cholinergic second pulses of paired thalamic stimuli. On average, the firing probabilities to the first and second pulses were similar

the initial excitation window, but was often followed by intense rebound firing (Fig. $7 A, B$, bottom trials of raster plots). In contrast, in trials with relatively long pauses, the pause was often preceded by one or more spikes in the initial excitation window, but was followed by little or no rebound firing (Fig. $7 A, B$, top trials of raster plots). When trials over all cholinergic interneurons were separated based on the number of spikes in the initial excitation phase and the mean PSTH recalculated for all trials and all neurons (cortex and thalamus stimulation combined for analysis), the initial excitation and rebound peaks in the PSTH scaled negatively (Fig. 7C). Accordingly, across cortical and thalamic responses in individual interneurons, the mean number of spikes fired in the initial excitation phase was positively correlated with the mean pause duration (Fig. 7D), but negatively correlated with the increase in firing during the rebound phase (Fig. 7E). The pause duration and rebound magnitude were not significantly correlated (Fig. 7F). Therefore, in summary, the initial excitation after stimulation of excitatory afferents was positively correlated with the duration of the pause, with more spikes resulting in a longer pause, and negatively correlated with the increase in firing during the rebound phase. The same phenomena could be observed in sorted raster plots of cholinergic interneurons responding to high-frequency train stimulation of cortex and thalamus (Fig. 7G,H). For high-frequency stimulation, the recalculated mean PSTH for trials with different numbers of spikes in the initial excitation phase (cortex and thalamus stimulation together) further indicated that the number of spikes in the initial excitation phase scaled positively with the pause length and negatively with the magnitude of the rebound (Fig. 7I). Notably, 
A

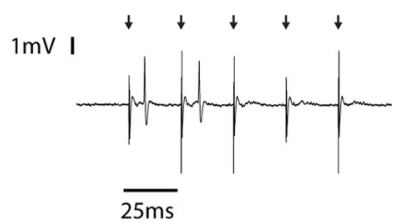

Ci

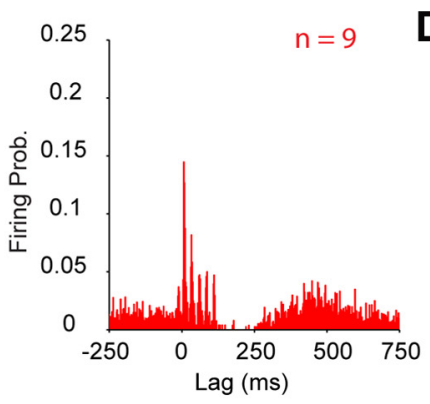

Cii

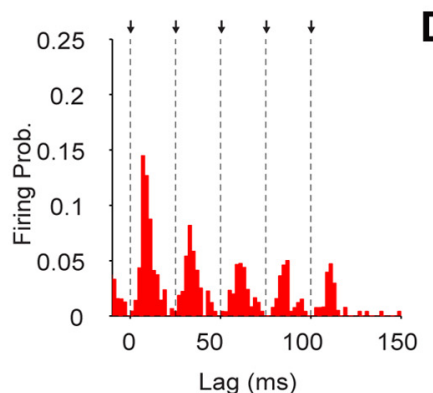

E

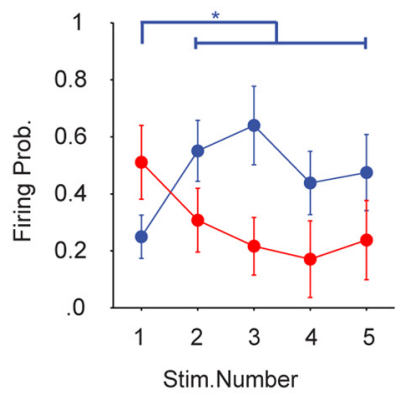

G

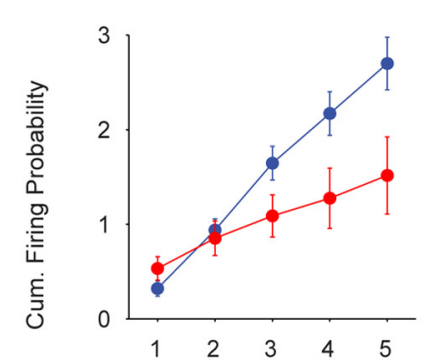

B

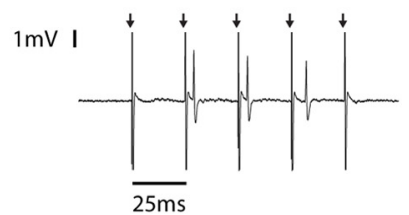

Di

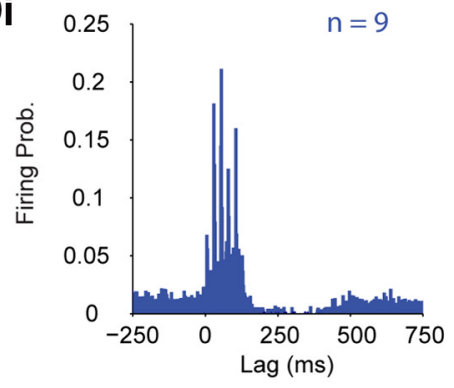

Dii

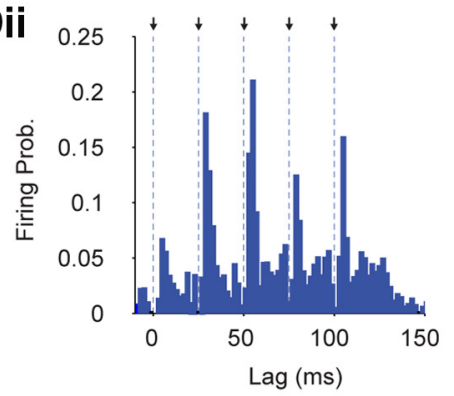

$\mathbf{F}$

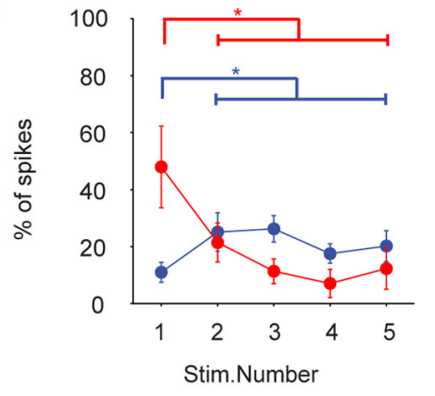

H

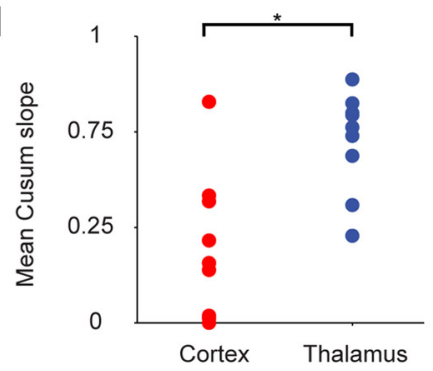

Figure 5. Differential responses of cholinergic interneurons to high-frequency stimulation of cortex and thalamus. $A, B$, Unit-activity recording (single representative sweep of stimulation artifacts and evoked responses, with arrows indicating stimulation onset) of a cholinergic interneuron that fired at short latency $(\sim 10 \mathrm{~ms})$ in response to $40 \mathrm{~Hz}$ trains $(5$ pulses $)$ of electrical stimuli delivered to the cortex $(\boldsymbol{A})$ or thalamus $(\boldsymbol{B})$. $\boldsymbol{C}$, Mean PSTH of all cholinergic interneurons $(n=9)$ that showed significant short-latency responses during $40 \mathrm{~Hz}$ cortical stimulation. Cii, Magnified view of $\mathbf{C}$ during the first $150 \mathrm{~ms}$ after the start of train stimulation. Di, Mean PSTH of all cholinergic interneurons $(n=9)$ that showed significant short-latency responses during $40 \mathrm{~Hz}$ thalamic stimulation. Dii, Magnified view of Diduring the first 150 ms after thestart of train stimulation. $E$, Mean firing probability of spikes fired at short latency on each pulse for cortical (red) and thalamic (blue) stimulation. The firing probability for the first thalamic pulse was significantly lower than the mean of the following four pulses (Wilcoxon signed-rank test, $p=0.004$ ). $\boldsymbol{F}$, Mean percentage of spikes fired on each pulse for cortical (red) and thalamic (blue) stimulation. The percentage of spikes fired after first thalamic pulse was significantly higher than the mean of the following four pulses (Wilcoxon signed-rank test, $p=0.027$ ), whereas for cortical stimulation, it was significantly lower (Wilcoxon signed-rank test, $p=0.027$ ). G, Mean cumulative sums (cusum) of firing of all interneurons in response to cortical (red) and thalamic (blue) stimulation over each pulse. $\boldsymbol{H}$, Slopes of the cusum for each interneuron (dots) to cortical and thalamic stimulation trains. The slope was significantly steeper for responses to thalamic stimulation (Mann-Whitney U test, $p=0.0078$ ).

and in contrast to similar experiments in vitro (Ding et al., 2010), high-frequency stimulation of cortex lead to pauses that were longer that the mean interspike interval (i.e., pause/interspike interval ratio of $>1$; Fig. $7 J)$. Across cortical and thalamic responses combined, the mean pause duration was positively correlated with the mean number of spikes in the initial excitation phase (Fig. 7J). For highfrequency stimulation, the increase in firing in the rebound phase was negatively correlated with both the mean spikes in the initial excitation (Fig. $7 \mathrm{~K}$ ) and the length of the pause (Fig. $7 L$ ). Together, these data show that the magnitude of the initial excitation of cholinergic interneurons was positively correlated with the length of their pauses and negatively correlated with the magnitude of their rebound firing.

\section{Multiphasic responses of tonically active neurons in behaving primates}

Our experiments in rats demonstrated that the magnitude of the initial excitation predicts the profile of the subsequent phases of the response in identified cholinergic interneurons. We next tested whether these relationships held true for putative cholinergic neurons recorded in behavioral situations. Therefore, we examined the multiphasic responses of TANs in four monkeys in relation to motivationally salient stimuli delivered in two different behavioral conditions: (1) a visual stimulus that preceded reward delivery by a fixed duration (the "rewardpredicting stimulus" condition; 79 TANs) and (2) the delivery of juice in the absence of any predictive cue (reward-only, 130 TANs). The major types of TAN response to motivationally salient stimuli were the same as those for identified cholinergic interneuron responses to afferent stimulation, although the prevalence of each response type varied across species (compare Figs. 6, 8). More specifically, in the reward-predicting stimulus condition, the mean response across all TANs clearly displayed all three phases: initial excitation, pause, and rebound (Fig. 8A). However, only $30 \%$ of individual TANs displayed all three phases in response to the reward-predicting stimulus in the PSTH (Fig. 8B, Cii), whereas the majority had only the pause and rebound phases (Fig. $8 B, C i$ ). In contrast, the mean response to reward-only had a relatively small initial excitation phase and prominent pause and rebound phases (Fig. $8 D$ ) and, accordingly, a smaller percentage (19\%) of TANs had all three response 
Single Stimulation.

High-Frequency Stimulation.
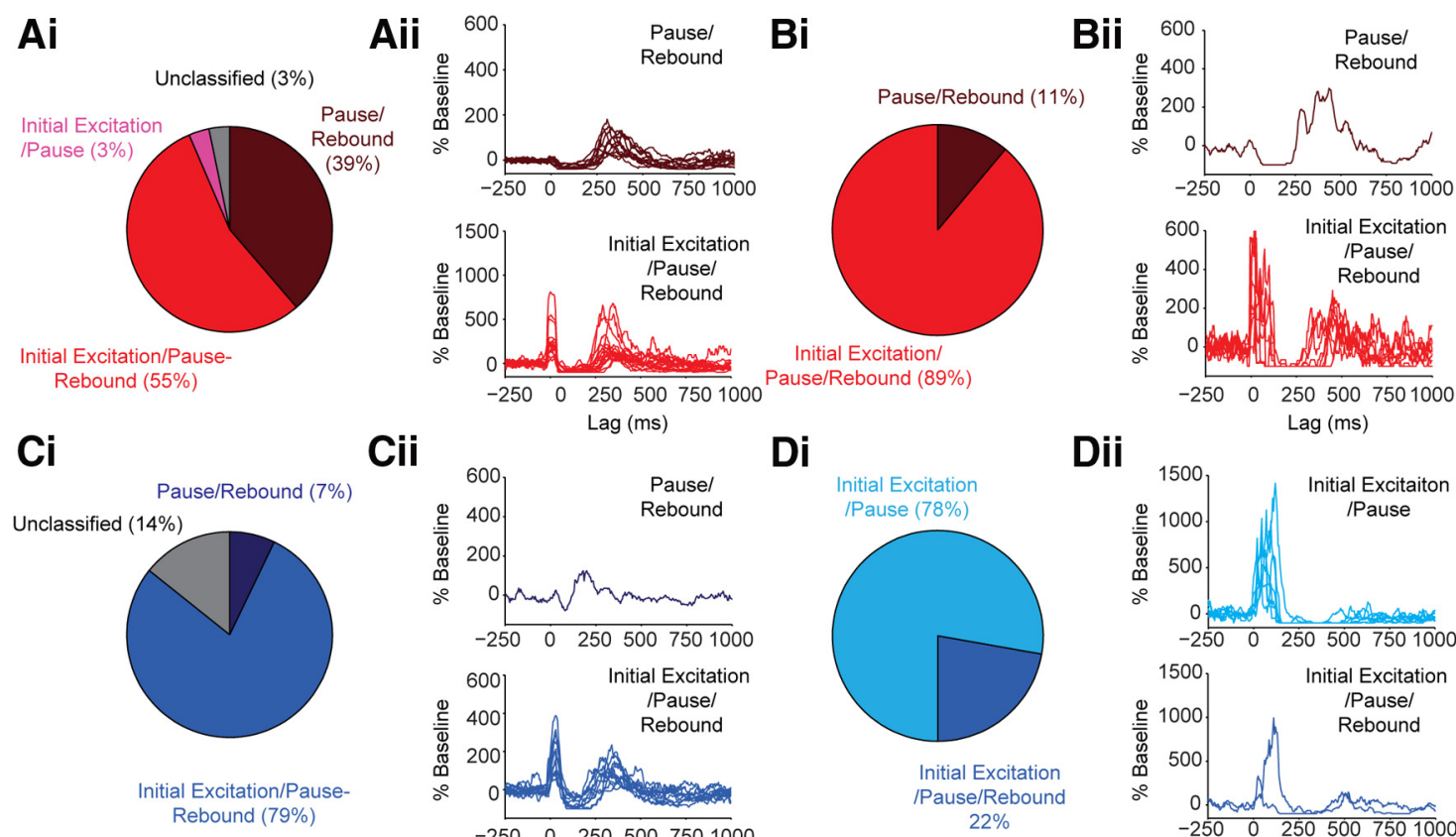

$\mathrm{Cii}$

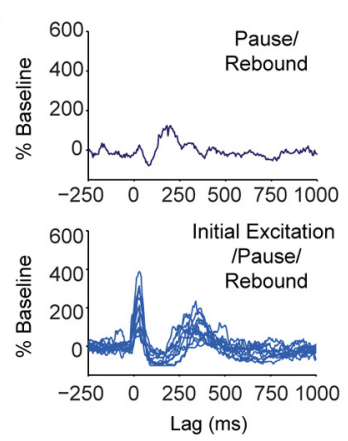

$\mathrm{Di}$

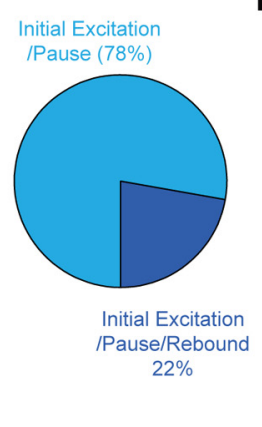

Dii

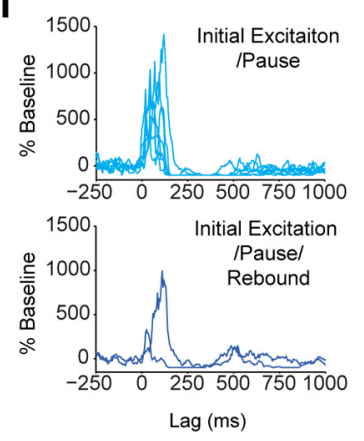

Figure 6. Multiphasic responses of cholinergic interneurons to stimulation of cortical and thalamic afferents. $\boldsymbol{A}, \boldsymbol{B}$, Classification of types of multiphasic responses to cortical stimulation. Ai, Percentage of different response types evoked by single-pulse cortical stimulation. Aii, PSTHs of all interneurons with the pause/rebound type of response (top) and the initial excitation/pause/ rebound type of response (bottom). Single stimuli were delivered at $0 \mathrm{~ms}$. Bi, Percentage of different response types evoked by a high-frequency cortical stimulation (train of 5 pulses at $40 \mathrm{~Hz}$ ). Bii, PSTHs of the one interneuron that displayed a pause/rebound response (top) and all the interneurons with initial excitation/pause/rebound responses (bottom). Start of train of high-frequency stimuli at $0 \mathrm{~ms}$. C, D, Classification of types of multiphasic responses to thalamic stimulation. Ci, Percentage of different responses types to single-pulse thalamic stimulation. Cii, PSTHs of the only interneuron with a pause/rebound response (top) and all the interneurons that displayed initial excitation/pause/rebound responses (bottom). Di, Percentage of different responses types evoked by a high-frequency thalamic stimulation. Dii, PSTHs of all interneurons with initial excitation/pause responses (top) and two neurons that displayed initial excitation/pause/rebound responses (bottom).

phases (Fig. 8 E, Fii). As for the reward-predicting stimulus, the majority of responses to reward-only were pause/rebound (Fig. $8 \mathrm{E}, \mathrm{Fi}$ ).

We then analyzed the different response phases of these monkey TANs using the same analytical framework as we used for quantifying the responses of rat interneurons to afferent stimulation (Fig. 7). For the reward-predicting stimulus, raster plots sorted by pause duration showed that, within the responses of single neurons, increased levels of spiking in the initial excitation window appeared to be followed by longer pauses and higher rebound firing regardless of whether there was a significant peak of initial excitation in the PSTH (Fig. 9A,B). When trials over all TANs were separated based on the number of spikes in the initial excitation phase and the mean PSTH recalculated, the magnitude of the rebound peak in the PSTH scaled negatively with the initial excitation magnitude (Fig. 9C). In this condition, the mean number of spikes in the initial excitation phase of each neuron was strongly positively correlated with the length of the pause phase (Fig. 9D). Moreover, the increase in firing rate in the rebound period was negatively correlated with the number of spikes in the initial excitation phase (Fig. $9 E$ ) and, to a lesser extent, with the pause length (Fig. 9F). This indicates that, in the behaving primate, the number of spikes fired shortly after presentation of a reward-predicting stimulus predicts the length and magnitude of the subsequent pause and rebound phases, respectively. We used a partial correlation analysis to assess whether the spontaneous firing rate could explain these relationships; for example, how much of the correlation between the initial excitation and pause/ rebound magnitude can be explained by their shared correlation with firing rate. All of the correlations above were still significant after partial correlation with the firing rate (Fig. 9D-F, values in parentheses), suggesting that it was not a confounding factor.

In contrast to the case of reward-predicting stimuli, PSTHs sorted by pause duration in the reward-only condition displayed no obvious relationship between the different response phases (Fig. 9G,H). When the population PSTH was plotted for all the trials with the same number of spikes in the initial excitation phase, there was no difference in the magnitude of the rebound phase (Fig. 9I). Although there was a significant positive correlation between the mean number of spikes in the initial excitation phase and the length of the pause, it was no longer significant after partial correlation with the firing rate (Spearman correlation coefficient, $p>0.05$ ). In addition, there were no significant correlations between the change in rate in the rebound phase and the mean number of spikes in the initial excitation or the length of the pause (Fig. $9 K, L$ ).

The mean latency of spikes in the initial excitation phase was significantly longer for the reward-only condition ( $89 \pm 5.7 \mathrm{~ms}$ ) compared with that in the reward-predicting stimulus condition (66.1 \pm 5 ms; Mann-Whitney test, $p=0.009)$. Because the onset of the initial excitation to reward-only was slower than that to the reward-predicting stimulus, which might partly arise because of the lower temporal precision of the unpredicted reward, the inclusion of spikes directly after presentation (before the true response) could have diluted the initial excitation phase and reduced the chances of significant correlations. We tested this hypothesis by running various analyses, such as excluding the first $30-50 \mathrm{~s}$ after stimulus presentation or by using fixed time 


\section{Single-pulse stimulation}

A
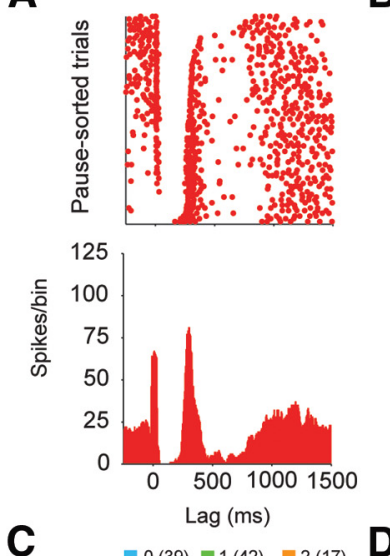

C $\quad \square 0(39) \backsim 1(42) \backsim 2(17)$
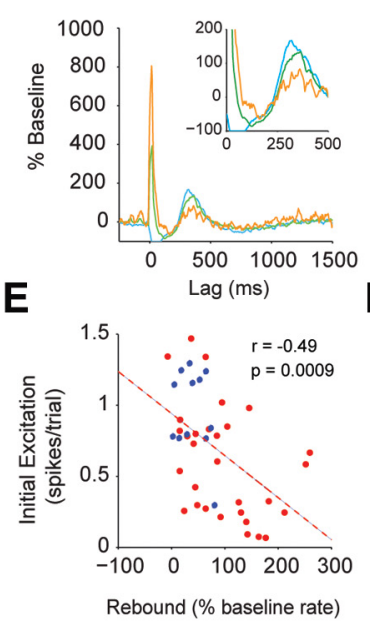

B
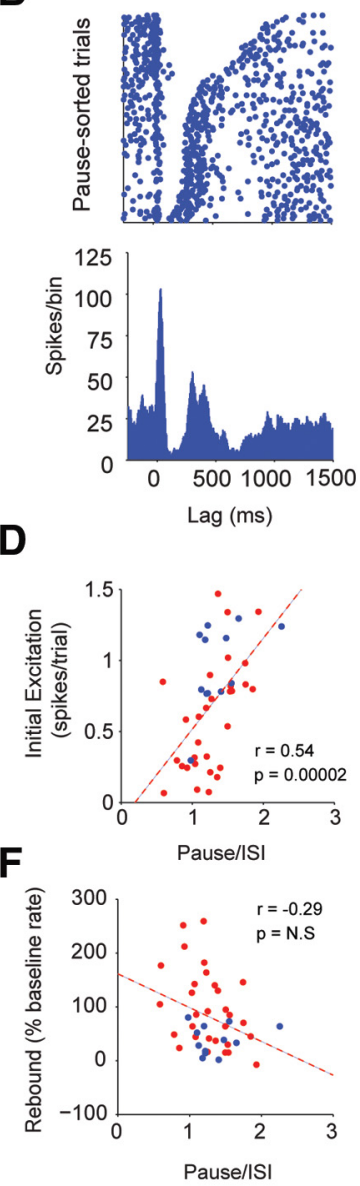

High-frequency stimulation

G
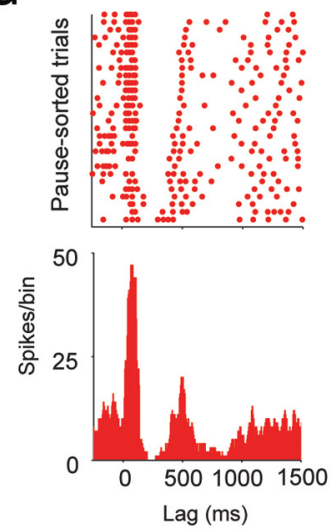

I
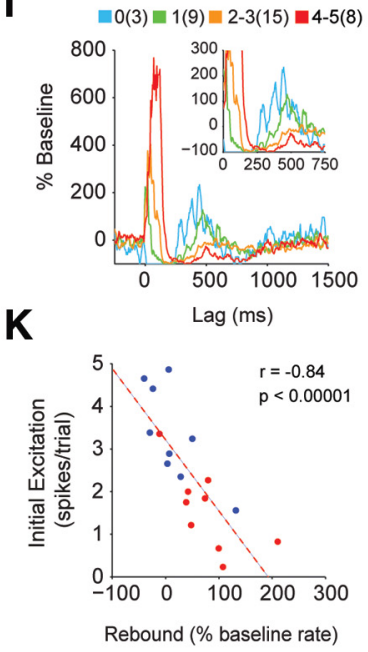

H
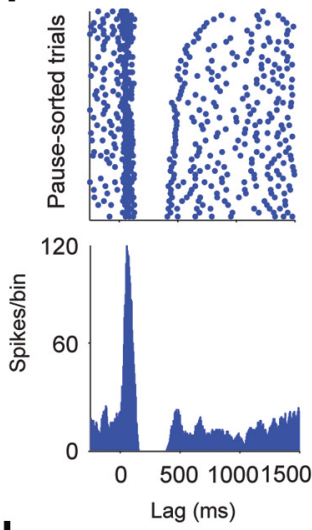

$J$
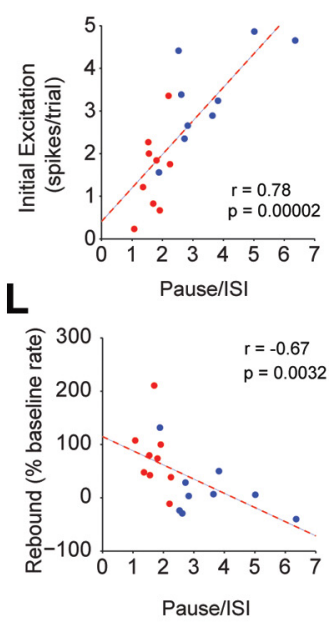

Figure 7. Initial excitation predicts the temporal profile of the multiphase response in cholinergic interneurons. $\boldsymbol{A}$, Raster plot with trials sorted by pause duration (top) and PSTH (bottom) for a cholinergic interneuron responding to single-pulse cortical stimulation. $\boldsymbol{B}$, As in $\boldsymbol{A}$, for the same neuron responding to single-pulse thalamic stimulation. $\boldsymbol{C}$, Mean PSTH across all responses (cortical and thalamic) sorted for the numbers of spikes in the initial excitation phase. Data from single-pulse stimulation of cortex and thalamus are combined. Responses were only included for a given neuron if there were $\geq 3$ trials with the requisite number of spikes in the initial excitation. Number of spikes in the initial excitation phase is shown using color code and the numbers of responses that were averaged are shown in parentheses. D, Scatter plot of the mean number of spikes fired in the initial excitation phase and the mean normalized pause length for all interneurons (dots) after single-pulse electrical stimulation of cortex (red) and thalamus (blue). The mean number of spikes in the initial excitation phase was positively correlated with the duration of the pause phase. Dotted line shows the best linear fit between the two variables. $\boldsymbol{E}, \boldsymbol{F}$, Change in firing rate in the rebound phase was significantly negatively correlated with the mean number of spikes in the initial excitation phase $(\boldsymbol{E})$, but not significantly correlated with the pause duration $(\boldsymbol{F}) . \boldsymbol{G}, \boldsymbol{H}$, Identical plots as $\boldsymbol{A}$ and $\boldsymbol{B}$ but for a different interneuron responding to high-frequency stimulation of cortex $(\boldsymbol{E})$ and thalamus $(\boldsymbol{F})$. I, Mean PSTHs across all trials with the same numbers of spikes in the intitial excitation phase for high-frequency stimulation of cortex and thalamus combined. Format as in $\boldsymbol{C}$. $\boldsymbol{J}$, Scatter plot of the mean number of spikes fired in the initial excitation phase and the mean normalized pause length for all neurons after cortical (red) and thalamic (blue) high-frequency electrical stimulation. The mean number of spikes in the initial excitation phase was positively correlated with the length of the pause phase across cortical and thalamic responses to stimulation. Dotted line shows the best linear fit between the two variables. $\boldsymbol{K}, \boldsymbol{L}$, The change in firing rate in the rebound phase was significantly negatively correlated with the mean number of spikes in the initial excitation phase $(\boldsymbol{K})$ and with the pause duration ( $\boldsymbol{L}$; format as in $\boldsymbol{J})$. Spearman correlation coefficients were used in all cases. NS, Not significant.

windows in relation only to the PSTH pause onset (e.g., counting all spikes $100 \mathrm{~ms}$ before the PSTH pause onset), rather than counting all the spikes after stimulus presentation to define the initial excitation phase (data not shown). These changes to the analysis did not lead to significant correlations between the initial excitation phase with the pause length (after partial correlation with firing rate) or rebound magnitude, suggesting that differences in the onset of the initial excitation did not account for the differences in response phase correlations across behavioral conditions.

Across all the TANs in both conditions, the values for the mean spikes in the initial excitation, pause length, and rebound rate increase were not significantly different between the rewardpredicting stimulus and reward-only conditions when calculated using trial-by-trial analysis (Mann-Whitney $\mathrm{U}$ test, $p>0.05$ ). Therefore, the main difference between the two conditions was the relationship between the different phases, rather than the durations/magnitudes of the individual phases. In summary, and as we observed in rat cholinergic neurons, the temporal profile of the multiphasic response of primate TANs could be predicted by the magnitude of the initial excitation. However, these correlations were only observed after reward-predicting stimuli, but were weak or absent in response to free reward delivery outside of a task context. Therefore, synaptic excitation by cortical and/or thalamic afferents helps explain the evolution of the multiphasic response in primate TANs under specific behavioral conditions.

\section{Discussion}

Cholinergic interneurons are key modulators of striatal microcircuit function. Their output is determined by the interplay of intrinsic membrane properties underlying their autonomous fir- 
Ai
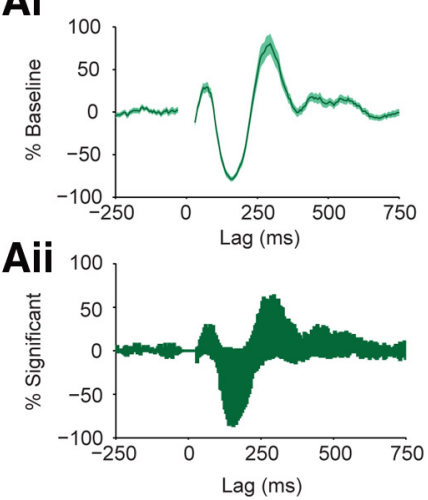

B

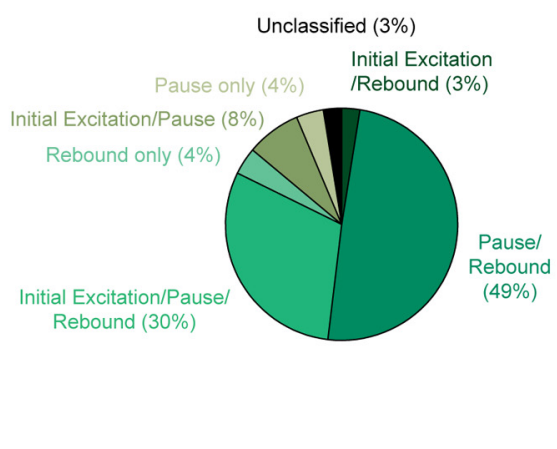

Reward-Only

Di

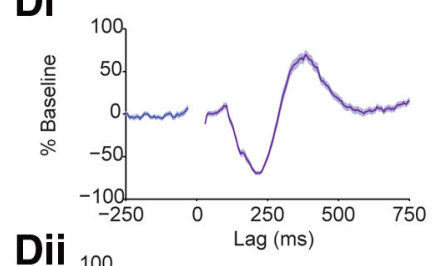

Dii

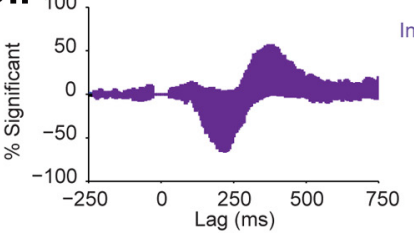

E
Reward-predicting stimulus
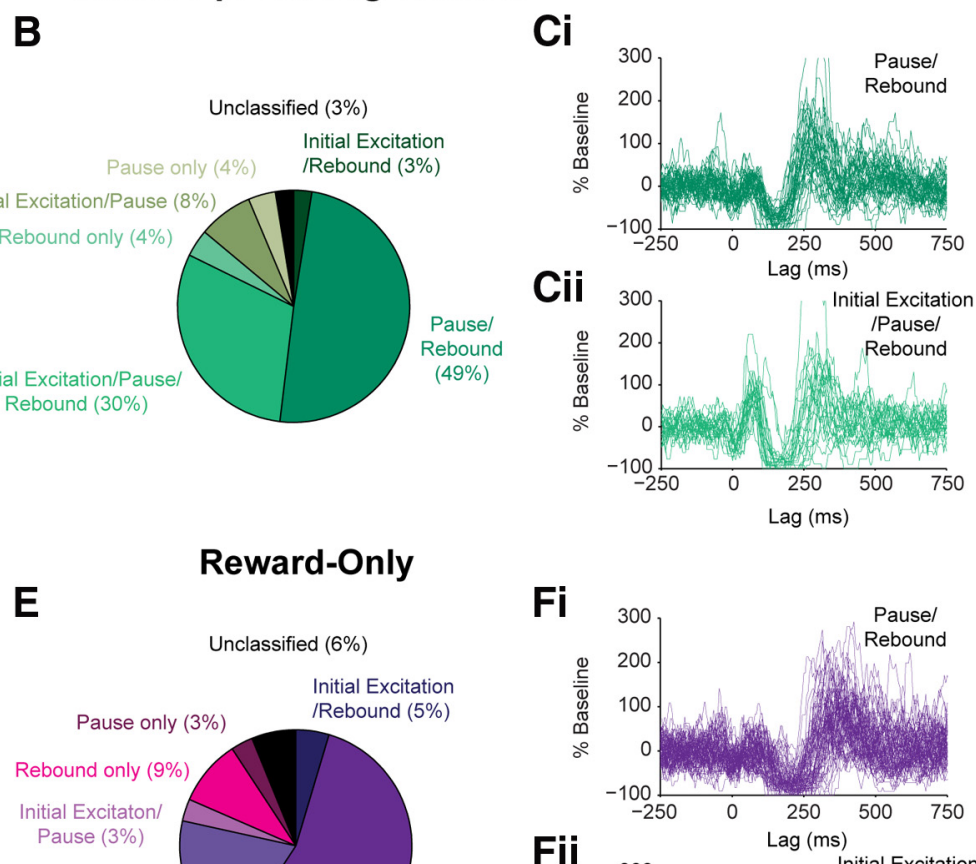

Fii

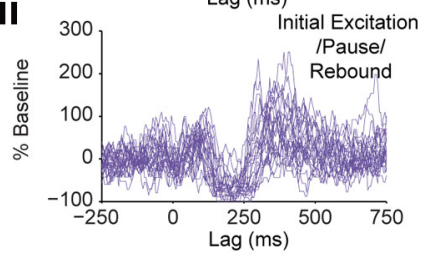

Figure 8. Multiphasic responses of primate TANs to motivationally salient stimuli. $\boldsymbol{A}$, Mean response (Ai) and the percentage of significant responses per bin (Aii) across the population of recorded TANs after the reward-predicting stimulus (given at $0 \mathrm{~ms}$ ). B, Classification of the types of multiphasic responses of all the TANs recorded in this first behavioral condition. C, PSTHs of all TANs with pause/rebound responses (Ci) and initial excitation/pause/rebound responses ( $(\boldsymbol{i} i)$ to the reward-predicting stimulus. $\boldsymbol{D}$, Mean response (Di) and the percentage of significant responses (Dii) across the population of recorded TANs after reward-only. E, Classification of multiphasic responses of all of the TANs recorded in this second behavioral condition. $F$, PSTHs of all TANs with pause/rebound responses (Fi) and initial excitation/pause/rebound responses (Fii) to reward-only.

ing (Bennett et al., 2000) and their synaptic inputs, which can alter spike rate and timing (Bennett and Wilson, 1998). Cholinergic interneurons commonly respond to motivationally salient stimuli with a pause in firing followed by a rebound increase in firing rate (Aosaki et al., 1994; Morris et al., 2004). Here, we demonstrate in the rat that the cortex and thalamus provide an initial excitation to identified cholinergic interneurons that can mediate the temporal profile of subsequent response phases and that, by extrapolation to TANs in monkeys, this influence is present and selective for behavioral condition.

One of the aims of this study was to quantify cortical and thalamic synaptic innervation of cholinergic interneurons. We found that an individual neuron can form synapses with cortical terminals and thalamic terminals, which can be with proximal dendrites. Overall, we found that thalamic terminals are more numerous than cortical terminals, which is consistent with previous studies (Lapper and Bolam, 1992). Despite attempts at controlling for antibody labeling, we cannot rule out the possibility that some of the terminals are false-negatives for the VGluT markers; however, it has been indicated that a proportion of terminals forming asymmetric synapses in the striatum are negative for both VGluT1 and VGluT2 (Lacey et al., 2005). This sparse excitatory, and in particular, cortical innervation does not mean that these inputs do not have significant effects, a point that is clear from the current and previous in vivo stimulation data (Wilson et al., 1990). Sparse inputs may have a particular role in regulating networks (Teramae et al., 2012).
Despite the apparently sparse nature of cortical inputs, we confirm that brief, single-pulse electrical stimulation of cortex often evokes short-latency spiking (Wilson et al., 1990; Reynolds and Wickens, 2004; Sharott et al., 2012). Thalamic inputs, and especially those arising from caudal ITN, are assumed to partly determine cholinergic interneuron activity in vivo (Schulz and Reynolds, 2013). We found that short-latency interneuron responses to matched stimulation of thalamus were similarly timed, but less robust compared with those driven by cortex. However, with each pulse of a high-frequency stimulus train, interneuron responses to thalamic inputs became increasingly robust, whereas responses to cortical inputs were progressively dampened. These contrasting response properties have important functional implications. For example, cortical and thalamic synapses with cholinergic interneurons might be subject to different short-term plasticity rules, at least in vivo (Ding et al., 2010). The mechanisms underlying this possible scenario are unknown. Nevertheless, attenuation of cortically-driven spiking with repeated input would constrain the influence of cortical inputs on short-latency acetylcholine release regardless of the strength of sustained input from a given cortical area. In contrast, spiking in response to salient/orienting inputs from the ITN would scale positively with the strength and duration of thalamic input. The short half-life of synaptically released acetylcholine, together with the fast kinetics of nicotinic acetylcholine receptors, would help to ensure accurate striatal "readout" of these distinct responses, which could support the important role of 


\section{Reward-predicting stimulus}

A
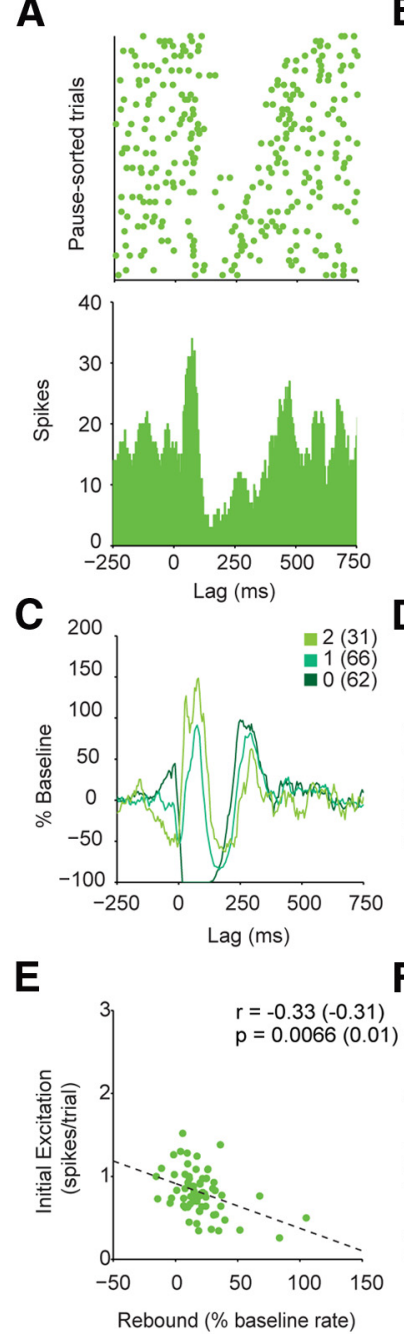

B
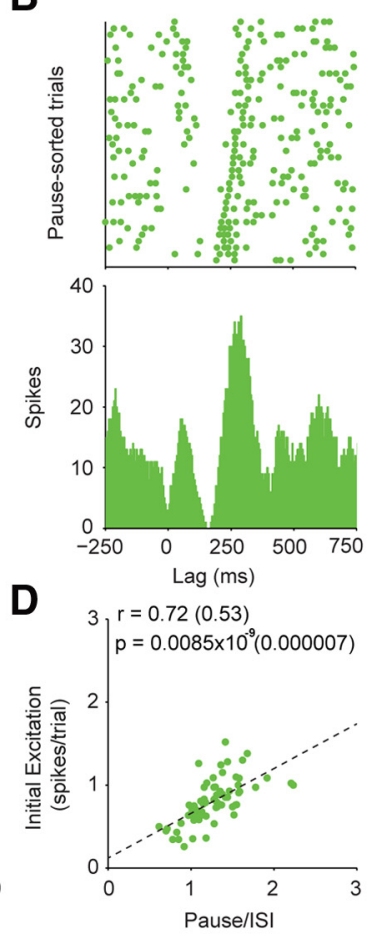

$\mathbf{F}$

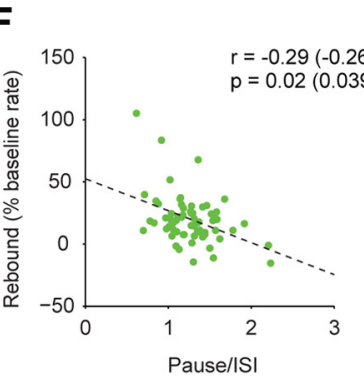

G
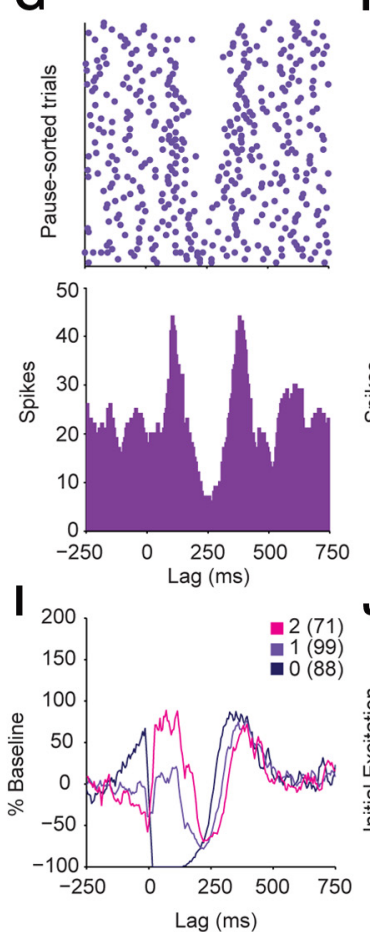

K

Reward-only

H
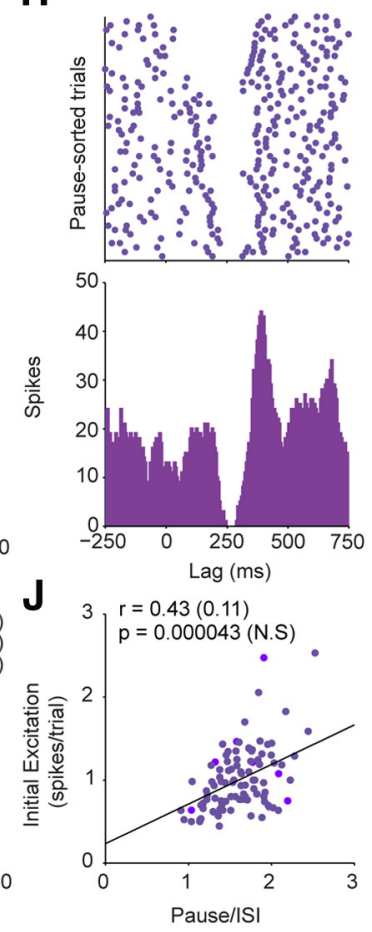

L

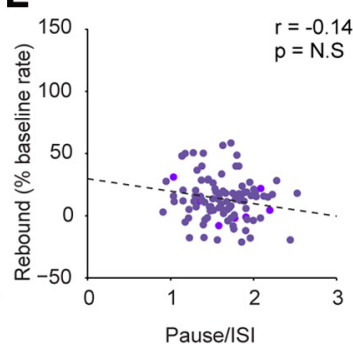

Figure 9. Dependence of multiphase responses of primate TANs on their initial excitation is behaviorally specific. $\boldsymbol{A}$, Raster plot with trials sorted by pause duration (top) and PSTH (bottom) for a TAN responding to the reward-predicting stimulus with an initial excitation/pause/rebound response. $\boldsymbol{B}$, Plots for a different TAN responding to the same condition with a pause/rebound response. C, Mean PSTHs for all trials with the same number of spikes in the initial excitation phase, in response to the reward-predicting stimulus. Responses were only included for a given TAN if there were $\geq 3$ trials with the requisite number of spikes in the initial excitation. Number of spikes in the initial excitation phase is shown using color code and the number of TANs that were averaged is shown in parenthesis. $\boldsymbol{D}$, Mean number of spikes in the initial excitation was positively correlated with the length of the pause phase across all neurons after partial correlation with firing rate (parentheses). $\boldsymbol{E}, \boldsymbol{F}$, Increase in firing rate in the rebound phase was significantly negatively correlated and the mean number of spikes in the initial excitation phase $(\boldsymbol{E})$ and with the length of the pause $(\boldsymbol{F})$. Both correlations were significant after partialization with firing rate (parentheses). G, Raster plot with trials sorted by pause duration (top) and PSTH (bottom) for a TAN responding to reward-only with an initial excitation/pause/rebound response. $\boldsymbol{H}$, Identical plots for a different TAN responding to the same condition with a pause/rebound response. $\boldsymbol{I}$, Mean PSTH for all neurons for trials with the same numbers of spikes in the initial excitation phase, in response to the unpredicted reward. Format as in $C . J$, Mean number of spikes in the initial excitation phase was correlated with the length of the pause phase across all neurons, but was no longer significant after partial correlation with firing rate (parentheses). $\boldsymbol{K}, \boldsymbol{L}$, Increase in firing rate in the rebound phase was not significantly correlated with the mean number of spikes in the initial excitation phase $(\boldsymbol{K})$ or with the length of the pause ( $\boldsymbol{L})$. Spearman correlation coefficients were used in all cases. NS, Not significant.

these thalamic inputs for learning and behavioral flexibility (Brown et al., 2010; Bradfield et al., 2013). Although cortical modulation of cholinergic interneurons has not been studied in behaving animals, diverse information carried by their cortical and thalamic afferents could play different roles in modulating their output and influence on behavior.

Importantly, we define a novel relationship between initial spiking and the multiphasic responses of cholinergic interneurons to stimulation of cortical/thalamic afferents. The two excitatory inputs did not produce specific multiphasic responses, but rather, the amount of initial excitation, regardless of the input source, predicted the temporal profile of the subsequent pause and rebound phases. However, because the properties of the initial excitation were different (cortex and thalamus being broadly depressing and facilitating, respectively), the nature of the multiphasic response was determined partly by its source. In the case of responses to cortical stimulation, the attenuation of shortlatency firing during high-frequency stimulation meant that rebound phases were the rule. Cortical afferents are therefore well suited to mediating a consistent pause length and a strong rebound. In contrast, sustained thalamic input favored more shortlatency spikes and, therefore, long pauses with little or no subsequent rebound. The nature of excitatory input can therefore determine acetylcholine release in striatum for hundreds of milliseconds after the short-latency modulation of spiking.

We also recorded the multiphasic responses of monkey TANs to motivationally salient stimuli and demonstrate that the correlations in initial spiking, pause, and rebound response phases as 
defined in rat hold true for primates under specific behavioral conditions. The pause in firing after rewarding stimuli has become another distinguishing physiological feature of TANs (Kimura et al., 1984; Apicella et al., 1991) and might provide a temporal framework for reinforcement learning (Morris et al., 2004). Importantly, the rebound phase scales with reward-based task performance (Apicella et al., 2011). We found that the number of spikes in the initial excitation phase was predictive of both the pause and rebound phases of the TAN response to a rewardpredicting stimulus. By extrapolating from our findings in rats, cortical and/or thalamic afferents emerge as key candidate drivers of the initial spiking phase of the responses of primate TANs to the reward-predicting stimulus. However, in the reward-only condition, correlations between TAN response phases were absent or weak, suggesting that responses to unpredicted reward might be underlain by different circuits/mechanisms. In both behavioral conditions, cholinergic transmission could modulate striatal dopamine release directly (Cachope et al., 2012; Threlfell et al., 2012) to further influence the processing of reward-based tasks. It will be important to test in future studies whether the principles defined here also apply to the ventral striatum.

A positive correlation between the initial spiking and pause duration of rodent interneurons has been shown in vitro (Oswald et al., 2009; Ding et al., 2010) and is a consequence of interactions between synaptic excitation and intrinsic currents. Indeed, excitatory inputs to these interneurons are reliably followed by an intrinsically generated afterhyperpolarization (AHP) that transiently suppresses their tonic firing (Oswald et al., 2009). Moreover, input magnitude and AHP duration are positively correlated (Oswald et al., 2009; Schulz et al., 2011). This mechanism likely explains the relationship between the initial excitation and pause phases of cholinergic interneurons in our stimulation experiments in rats and could also underlie the same response phases of TANs after reward-predicting stimuli. Conversely, an unambiguous dissection of whether and how rebound firing might emerge from synaptic excitation-AHP sequences is lacking (Schulz and Reynolds, 2013), a scenario compounded by the absence of rebound interneuron firing after afferent stimulation in previous experiments in vivo and in vitro (Oswald et al., 2009; Ding et al., 2010; Schulz et al., 2011). One proposal is that the rebound reflects a separate and subsequent excitation from cortex and/or thalamus (Schulz and Reynolds, 2013). A specialized population of ITN cells that respond at long latencies to orienting stimuli could subserve this late excitation (Matsumoto et al., 2001; Schulz and Reynolds, 2013). The co-release of glutamate by cholinergic interneurons (Higley et al., 2011) might also shape their multiphasic responses through feedforward excitation. However, this seems unlikely for some relationships defined here; for example, the initial excitation and rebound phases were negatively correlated. Overall, our findings support the possibility that a brief excitatory drive can initiate a sequence of intrinsic and/or local circuit events that trigger both the pause and the rebound, but in a way that ensures that these two response phases are negatively correlated.

The correlations between TAN response phases were clear after reward-predicting stimuli but not after unpredicted rewards. This raises the possibility that the two behavioral conditions recruited distinct sets of inputs to differentially influence firing. We found that $\sim 60 \%$ of synapses made are symmetric, providing further evidence that GABAergic inputs likely play crucial roles in modulating these cells. Indeed, inhibition of cholinergic interneurons by optogenetic stimulation of their GABAergic inputs can produce rebound responses (English et al.,
2011; Brown et al., 2012). Therefore, the pause in the reward-only condition has several possible sources of GABAergic inhibition, such as axon collaterals of MSNs (Gonzales et al., 2013), GABAergic striatal interneurons (Sullivan et al., 2008; Gonzales et al., 2013), and GABAergic projection neurons in the external globus pallidus (Mallet et al., 2012) and midbrain (Brown et al., 2012). The idea that inputs from midbrain could mediate TAN responses to reward would fit with the role of these distal structures in processing reward-related stimuli. Moreover, the intrinsic membrane properties of cholinergic interneurons homogenize the length of their pauses to hyperpolarizing inputs of varying strength (Wilson, 2005), which could disassociate the strength of inhibitory inputs from the magnitude of the rebound.

Elucidating how the dynamic spiking of striatal cholinergic interneurons is governed by their myriad synaptic inputs is critical for fully understanding their functional roles in striatal microcircuits and contributions to adaptive behavior. With this mind, we conclude that cortex and thalamus can provide an initial synaptic excitation that shapes the multiphasic responses of these interneurons to motivationally salient stimuli under specific behavioral conditions.

\section{References}

Adler A, Finkes I, Katabi S, Prut Y, Bergman H (2013a) Encoding by synchronization in the primate striatum. J Neurosci 33:4854-4866. CrossRef Medline

Adler A, Katabi S, Finkes I, Prut Y, Bergman H (2013b) Different correlation patterns of cholinergic and GABAergic interneurons with striatal projection neurons. Front Syst Neurosci 7:47. CrossRef Medline

Aosaki T, Tsubokawa H, Ishida A, Watanabe K, Graybiel AM, Kimura M (1994) Responses of tonically active neurons in the primate's striatum undergo systematic changes during behavioral sensorimotor conditioning. J Neurosci 14:3969-3984. Medline

Apicella P (2007) Leading tonically active neurons of the striatum from reward detection to context recognition. Trends Neurosci 30:299-306. CrossRef Medline

Apicella P, Scarnati E, Schultz W (1991) Tonically discharging neurons of monkey striatum respond to preparatory and rewarding stimuli. Exp Brain Res 84:672-675. Medline

Apicella P, Legallet E, Trouche E (1997) Responses of tonically discharging neurons in the monkey striatum to primary rewards delivered during different behavioral states. Exp Brain Res 116:456-466. CrossRef Medline

Apicella P, Ravel S, Deffains M, Legallet E (2011) The role of striatal tonically active neurons in reward prediction error signaling during instrumental task performance. J Neurosci 31:1507-1515. CrossRef Medline

Bennett BD, Wilson CJ (1998) Synaptic regulation of action potential timing in neostriatal cholinergic interneurons. J Neurosci 18:8539-8549. Medline

Bennett BD, Callaway JC, Wilson CJ (2000) Intrinsic membrane properties underlying spontaneous tonic firing in neostriatal cholinergic interneurons. J Neurosci 20:8493-8503. Medline

Bolam JP, ed (1992) Experimental neuroanatomy. Oxford: OUP.

Bradfield LA, Bertran-Gonzalez J, Chieng B, Balleine BW (2013) The thalamostriatal pathway and cholinergic control of goal-directed action: interlacing new with existing learning in the striatum. Neuron 79:153166. CrossRef Medline

Brown HD, Baker PM, Ragozzino ME (2010) The parafascicular thalamic nucleus concomitantly influences behavioral flexibility and dorsomedial striatal acetylcholine output in rats. J Neurosci 30:14390-14398. CrossRef Medline

Brown MT, Tan KR, O'Connor EC, Nikonenko I, Muller D, Lüscher C (2012) Ventral tegmental area GABA projections pause accumbal cholinergic interneurons to enhance associative learning. Nature 492:452456. CrossRef Medline

Cachope R, Mateo Y, Mathur BN, Irving J, Wang HL, Morales M, Lovinger DM, Cheer JF (2012) Selective activation of cholinergic interneurons enhances accumbal phasic dopamine release: setting the tone for reward processing. Cell Rep 2:33-41. CrossRef Medline

Deffains M, Legallet E, Apicella P (2010) Modulation of neuronal activity in 
the monkey putamen associated with changes in the habitual order of sequential movements. J Neurophysiol 104:1355-1369. CrossRef Medline

Dimova R, Vuillet J, Nieoullon A, Kerkerian-Legoff L (1993) Ultrastructural features of the choline acetyltransferase-containing neurons and relationships with nigral dopaminergic and cortical afferent pathways in the rat striatum. Neuroscience 53:1059-1071. CrossRef Medline

Ding JB, Guzman JN, Peterson JD, Goldberg JA, Surmeier DJ (2010) Thalamic gating of corticostriatal signaling by cholinergic interneurons. Neuron 67:294-307. CrossRef Medline

Doig NM, Moss J, Bolam JP (2010) Cortical and thalamic innervation of direct and indirect pathway medium-sized spiny neurons in mouse striatum. J Neurosci 30:14610-14618. CrossRef Medline

Dubé L, Smith AD, Bolam JP (1988) Identification of synaptic terminals of thalamic or cortical origin in contact with distinct medium-size spiny neurons in the rat neostriatum. J Comp Neurol 267:455-471. CrossRef Medline

English DF, Ibanez-Sandoval O, Stark E, Tecuapetla F, Buzsáki G, Deisseroth K, Tepper JM, Koos T (2011) GABAergic circuits mediate the reinforcement-related signals of striatal cholinergic interneurons. Nat Neurosci 15:123-130. CrossRef Medline

Gonzales KK, Pare JF, Wichmann T, Smith Y (2013) GABAergic inputs from direct and indirect striatal projection neurons onto cholinergic interneurons in the primate putamen. J Comp Neurol 521:2502-2522. CrossRef Medline

Henny P, Brown MT, Northrop A, Faunes M, Ungless MA, Magill PJ, Bolam JP (2012) Structural correlates of heterogeneous in vivo activity of midbrain dopaminergic neurons. Nat Neurosci 15:613-619. CrossRef Medline

Henny P, Brown MT, Micklem BR, Magill PJ, Bolam JP (2013) Stereological and ultrastructural quantification of the afferent synaptome of individual neurons. Brain Struct Funct, in press. CrossRef

Higley MJ, Gittis AH, Oldenburg IA, Balthasar N, Seal RP, Edwards RH, Lowell BB, Kreitzer AC, Sabatini BL (2011) Cholinergic interneurons mediate fast VGluT3-dependent glutamatergic transmission in the striatum. PLoS One 6:e19155. CrossRef Medline

Inokawa H, Yamada H, Matsumoto N, Muranishi M, Kimura M (2010) Juxtacellular labeling of tonically active neurons and phasically active neurons in the rat striatum. Neuroscience 168:395-404. CrossRef Medline

Jiao Y, Sun Z, Lee T, Fusco FR, Kimble TD, Meade CA, Cuthbertson S, Reiner A (1999) A simple and sensitive antigen retrieval method for freefloating and slide-mounted tissue sections. J Neurosci Methods 93:149162. CrossRef Medline

Joshua M, Adler A, Mitelman R, Vaadia E, Bergman H (2008) Midbrain dopaminergic neurons and striatal cholinergic interneurons encode the difference between reward and aversive events at different epochs of probabilistic classical conditioning trials. J Neurosci 28:11673-11684. CrossRef Medline

Kimura M, Rajkowski J, Evarts E (1984) Tonically discharging putamen neurons exhibit set-dependent responses. Proc Natl Acad Sci U S A 81: 4998-5001. CrossRef Medline

Kusnoor SV, Parris J, Muly EC, Morgan JI, Deutch AY (2010) Extracerebellar role for Cerebellin1: modulation of dendritic spine density and synapses in striatal medium spiny neurons. J Comp Neurol 518:2525-2537. CrossRef Medline

Lacey CJ, Boyes J, Gerlach O, Chen L, Magill PJ, Bolam JP (2005) GABA(B) receptors at glutamatergic synapses in the rat striatum. Neuroscience 136: 1083-1095. CrossRef Medline

Lapper SR, Bolam JP (1992) Input from the frontal-cortex and the parafascicular nucleus to cholinergic interneurons in the dorsal striatum of the rat. Neuroscience 51:533-545. CrossRef Medline

Llinas RR, Leznik E, Urbano FJ (2002) Temporal binding via cortical coincidence detection of specific and nonspecific thalamocortical inputs: a voltage-dependent dye-imaging study in mouse brain slices. Proc Natl Acad Sci U S A 99:449-454. CrossRef Medline

Magill PJ, Bolam JP, Bevan MD (2000) Relationship of activity in the subthalamic nucleus-globus pallidus network to cortical electroencephalogram. J Neurosci 20:820-833. Medline

Magill PJ, Sharott A, Bevan MD, Brown P, Bolam JP (2004) Synchronous unit activity and local field potentials evoked in the subthalamic nucleus by cortical stimulation. J Neurophysiol 92:700-714. CrossRef Medline

Magill PJ, Pogosyan A, Sharott A, Csicsvari J, Bolam JP, Brown P (2006) Changes in functional connectivity within the rat striatopallidal axis dur- ing global brain activation in vivo. J Neurosci 26:6318-6329. CrossRef Medline

Mallet N, Le Moine C, Charpier S, Gonon F (2005) Feedforward inhibition of projection neurons by fast-spiking GABA interneurons in the rat striatum in vivo. J Neurosci 25:3857-3869. CrossRef Medline

Mallet N, Pogosyan A, Márton LF, Bolam JP, Brown P, Magill PJ (2008) Parkinsonian beta oscillations in the external globus pallidus and their relationship with subthalamic nucleus activity. J Neurosci 28:1424514258. CrossRef Medline

Mallet N, Micklem BR, Henny P, Brown MT, Williams C, Bolam JP, Nakamura KC, Magill PJ (2012) Dichotomous organization of the external globus pallidus. Neuron 74:1075-1086. CrossRef Medline

Matsumoto N, Minamimoto T, Graybiel AM, Kimura M (2001) Neurons in the thalamic CM-Pf complex supply striatal neurons with information about behaviorally significant sensory events. J Neurophysiol 85:960976. Medline

Morris G, Arkadir D, Nevet A, Vaadia E, Bergman H (2004) Coincident but distinct messages of midbrain dopamine and striatal tonically active neurons. Neuron 43:133-143. CrossRef Medline

Nanda B, Galvan A, Smith Y, Wichmann T (2009) Effects of stimulation of the centromedian nucleus of the thalamus on the activity of striatal cells in awake rhesus monkeys. Eur J Neurosci 29:588-598. CrossRef Medline

Oswald MJ, Oorschot DE, Schulz JM, Lipski J, Reynolds JN (2009) IH current generates the afterhyperpolarisation following activation of subthreshold cortical synaptic inputs to striatal cholinergic interneurons. J Physiol 587:5879-5897. CrossRef Medline

Paxinos G, Watson C (1986) The rat brain in stereotaxic coordinates, Ed 2. Sydney: Academic.

Ravel S, Legallet E, Apicella P (2003) Responses of tonically active neurons in the monkey striatum discriminate between motivationally opposing stimuli. J Neurosci 23:8489-8497. Medline

Reynolds JN, Wickens JR (2004) The corticostriatal input to giant aspiny interneurons in the rat: a candidate pathway for synchronising the response to reward-related cues. Brain Res 1011:115-128. CrossRef Medline

Sadek AR, Magill PJ, Bolam JP (2007) A single-cell analysis of intrinsic connectivity in the rat globus pallidus. J Neurosci 27:6352-6362. CrossRef Medline

Schulz JM, Reynolds JN (2013) Pause and rebound: sensory control of cholinergic signaling in the striatum. Trends Neurosci 36:41-50. CrossRef Medline

Schulz JM, Oswald MJ, Reynolds JN (2011) Visual-induced excitation leads to firing pauses in striatal cholinergic interneurons. J Neurosci 31:1113311143. CrossRef Medline

Sharott A, Doig NM, Mallet N, Magill PJ (2012) Relationships between the firing of identified striatal interneurons and spontaneous and driven cortical activities in vivo. J Neurosci 32:13221-13236. CrossRef Medline

Sizemore RJ, Reynolds JN, Oorschot DE (2010) Number and type of synapses on the distal dendrite of a rat striatal cholinergic interneuron: a quantitative, ultrastructural study. J Anat 217:223-235. CrossRef Medline

Somogyi P, Bolam JP, Smith AD (1981) Monosynaptic cortical input and local axon collaterals of identified striatonigral neurons. A light and electron microscopic study using the Golgi-peroxidase transportdegeneration procedure. J Comp Neurol 195:567-584. CrossRef Medline

Sullivan MA, Chen H, Morikawa H (2008) Recurrent inhibitory network among striatal cholinergic interneurons. J Neurosci 28:8682-8690. CrossRef Medline

Teramae JN, Tsubo Y, Fukai T (2012) Optimal spike-based communication in excitable networks with strong-sparse and weak-dense links. Sci Rep 2:485. CrossRef Medline

Threlfell S, Lalic T, Platt NJ, Jennings KA, Deisseroth K, Cragg SJ (2012) Striatal dopamine release is triggered by synchronized activity in cholinergic interneurons. Neuron 75:58-64. CrossRef Medline

Wilson CJ (2005) The mechanism of intrinsic amplification of hyperpolarizations and spontaneous bursting in striatal cholinergic interneurons. Neuron 45:575-585. CrossRef Medline

Wilson CJ, Chang HT, Kitai ST (1990) Firing patterns and synaptic potentials of identified giant aspiny interneurons in the rat neostriatum. J Neurosci 10:508-519. Medline 